\title{
Xây dựng độ đo thuần nhất và nâng cao độ tương phản của ảnh màu theo tiếp cận trực tiếp dựa trên đại số gia tử
}

\author{
Nguyễn Văn Quyền ${ }^{1}$, Ngô Hoàng Huy ${ }^{2}$, Nguyễn Cát Hồ ${ }^{3}$, Trần Thái Sơn ${ }^{2}$ \\ 1. Trường Đại học Hải Phòng \\ 2. Viện Công nghệ Thông tin, Viện Hàn lâm Khoa học và Công nghệ Việt Nam \\ 3. Trung tâm Nghiên cứu và Phát triển, Trường Đại học Duy Tân \\ E-mail: quyenqlkh.dhhp@gmail.com, huyngo3i@gmail.com, ncatho@gmail.com, ttson@ioit.ac.vn \\ Tác giả liên hệ: Nguyễn Văn Quyền \\ Ngày nhận: 10/04/2017, ngày sửa chữa: 08/09/2017, ngày duyệt đăng: 25/10/2017
}

Tóm tắt: Các kỹ thuật nâng cao tương phản ảnh đã thu hút nhiều sự quan tâm của cộng đồng xử lý ảnh vì chúng giúp cải thiện khả năng phân tích hình ảnh, thị lực máy tính, v.v. Trong các phương pháp nâng cao độ tương phản ảnh, các phương pháp trực tiếp thiết lập một độ đo tương phản và nâng cao chất lượng hình ảnh bằng cách cải thiện độ đo tương phản. Tuy nhiên, hiện có rất ít các nghiên cứu theo phương pháp trực tiếp, chỉ có các nghiên cứu của H.-D. Cheng và $\mathrm{H}$. Xu là đề xuất một phương pháp thay đổi độ tương phản tại mỗi điểm ảnh sử dụng độ đo tương phản được tính dựa trên độ thuần nhất của điểm ảnh. Trong bài báo này, chúng tôi đề xuất một phương pháp xây dựng độ thuần nhất mới dựa trên đại số gia tử. Các kết quả thực nghiệm cho thấy phương pháp nâng cao độ tương phản trực tiếp được thực hiện tốt với nhiều loại ảnh màu khác nhau khi áp dụng phép đo độ thuần nhất đề xuất.

Từ khóa: Nâng cao độ tương phản ảnh trực tiếp, độ đo thuần nhất, độ đo tưong phản, đại số gia tử.

Title: A New Homogeneity Measure Construction for Color Image Direct Contrast Enhancement based on Hedge Algebra

Abstract: Image contrast enhancement has attracted much attention of the image processing community because they help improve the image interpretability or visualization or bring out useful information hidden in image details. Among different methods, direct contrast enhancement establishes a contrast measure and then enhances the image quality by improving the contrast measure. However, there exist few studies of direct contrast enhancement, apart from those by H.-D. Cheng and $\mathrm{H} . \mathrm{Xu}$ in which a method was proposed to modify the contrast at each pixel, using a contrast measure of the pixel calculated from the homogeneity measure of the pixel. In this paper we propose a method to construct a new homogeneity measure based on hedge algebra. Experimental results demonstrated that direct contrast enhancement performs well for various color images when applied together with the proposed homogeneity measure.

Keywords: Direct contrast enhancement, homogeneity measure, contrast measure, hedge algebra.

\section{GIỚI THIỆU}

Nâng cao độ tương phản của ảnh là một vấn đề quan trọng trong xử lý và phân tích hình ảnh, là một bước cơ bản trong phân đoạn ảnh. Các kỹ thuật thông dụng để nâng cao độ tương phản của ảnh được phân loại theo hai tiếp cận chính: 1) các phương pháp gián tiếp và 2) các phương pháp trực tiếp $[1,2]$. Có nhiều kỹ thuật đã được đề xuất trong [3-8], hầu hết trong số đó là phương pháp gián tiếp. Các phương pháp này biến đổi histogram mà không sử dụng bất kỳ một độ đo tương phản (contrast measure) nào. Mặc dù vậy, có rất ít các nghiên cứu theo phương pháp trực tiếp trong đó biến đổi độ tương phản của ảnh dựa trên một độ đo tương phản xác định tại mỗi điểm ảnh, chẳng hạn các nghiên cứu [1, 2, 9-11].

Cho đến nay, hầu như chỉ có các nghiên cứu của $\mathrm{H}$.D. Cheng và $\mathrm{H}$. Xu $[1,2]$ là đề xuất một phương pháp biến đổi độ tương phản tại mỗi điểm ảnh dựa trên định nghĩa độ đo tương phản giữa độ sáng điểm ảnh và lân cận xung quanh nó. Độ đo tương phản của $[1,2]$ được xây dựng qua ba bước cơ bản. Thứ nhất, các đặc trưng địa phương như gradient, entropy, độ lệch chuẩn trung bình và moment bậc 4 tại từng điểm ảnh được tính và kết nhập thành một 
Bảng I

CÁC KÝ HIỆU VÀ CÁC ĐỊNH NGHĨA CỦA NÓ

\begin{tabular}{|c|c|}
\hline Ký hiệu & Định nghĩa \\
\hline$I$ & Ảnh RGB nói chung \\
\hline$M, N$ & $M \times N$ là kích thước theo pixel của ảnh đầu vào \\
\hline$I_{\mathrm{R}}, I_{\mathrm{G}}, I_{\mathrm{B}}$ & $\begin{array}{l}\text { Kênh ảnh } R, G \text { và } B \text { của ảnh màu trong biểu diễn màu } \\
\text { RGB }\end{array}$ \\
\hline $\begin{array}{l}I_{\mathrm{S}}, I_{\mathrm{H}} \\
I_{\mathrm{V}}\end{array}$ & $\begin{array}{l}\text { Kênh ảnh } \mathrm{H}, \mathrm{S} \text { và } \mathrm{V} \text { của ảnh màu trong biểu diễn màu } \\
\text { HSV }\end{array}$ \\
\hline $\begin{array}{l}L_{k, \min } \\
L_{k, \max }\end{array}$ & $\begin{array}{l}\text { Miền giá trị mức xám của kênh ảnh thứ } k \text {, ảnh đầu } \\
\text { vào, thông thường } L_{k, \min }=0, L_{k, \max }=255\end{array}$ \\
\hline $\mathrm{D}$ & $d \times d$ là kích thước cửa sổ lân cận của điểm ảnh \\
\hline$E_{i j}$ & $\begin{array}{l}\text { Giá trị gradient lấy tại điểm ảnh }(i, j) \text { được chuẩn hóa } \\
\text { về miền }[0,1] \text { theo một toán tử tìm kiếm biên chẳng } \\
\text { hạn toán tử Sobel }\end{array}$ \\
\hline$H_{i j}$ & $\begin{array}{l}\text { Giá trị entropy địa phương lấy tại điểm ảnh }(i, j) \text { được } \\
\text { chuẩn hóa về miền }[0,1]\end{array}$ \\
\hline$V_{i j}$ & $\begin{array}{l}\text { Độ lệch chuẩn trung bình mức xám lấy tại điểm ảnh } \\
(i, j) \text { được chuẩn hóa về miền }[0,1]\end{array}$ \\
\hline$R_{4, i j}$ & $\begin{array}{l}\text { Giá trị moment bầc } 4 \text { lấy tại điểm ảnh }(i, j) \text { được } \\
\text { chuẩn hóa về miền }[0,1]\end{array}$ \\
\hline$H O_{i j}$ & Giá trị kết nhập dạng $f\left(E_{i j}, H_{i j}, V_{i j}, R_{4, i j}\right)[11]$ \\
\hline$\beta_{i j}$ & Giá trị thuần nhất tại điểm ảnh $(i, j)$ \\
\hline$\delta_{i j}$ & Giá trị mức xám không thuần nhất tại điểm ảnh $(i, j)$ \\
\hline$\xi_{i j}$ & Số mũ khuếch đại tại $(i, j)$ \\
\hline $\mathrm{T}$ & $\in(0,1)$ : Tham số của phép nâng độ khuếch đại \\
\hline$f_{1}, f_{2}$ & $\in(0,1)$ : Tham số xác định dải động mức xám [11] \\
\hline$K$ & Số kênh ảnh cần xử lý của ảnh đầu vào \\
\hline$C$ & Số cụm cần phân cụm của tổ hợp kênh ảnh đầu vào \\
\hline$\mu_{i, j, c}$ & $\begin{array}{l}\text { Giá trị độ thuộc cụm thứ } c \text { của điểm ảnh }(i, j) \text {, đầu ra } \\
\text { của thủ tục phân cụm FCM }\end{array}$ \\
\hline$f_{\text {cut }}$ & $\begin{array}{l}\in \quad(0,1) \text { : Tham số xác định } C \text { dải động mức xám của } \\
\text { một kênh ảnh (mục III) }\end{array}$ \\
\hline
\end{tabular}

giá trị chỉ mức độ thuần nhất của điểm ảnh. Thứ hai, từ các giá trị độ thuần nhất của điểm ảnh các tác giả định nghĩa mức độ sáng xung quanh điểm ảnh, được gọi là giá trị trung bình không thuần nhất của điểm ảnh. Thứ ba, tính độ tương phản giữa mức sáng của điểm ảnh và giá trị trung bình không thuần nhất của điểm ảnh.

Chất lượng ảnh được nâng cao độ tương phản phụ thuộc vào giá trị thuần nhất tại mỗi điểm ảnh, bởi vì độ đo thuần nhất liên quan chính đến các thông tin địa phương của một ảnh và phản ánh tính đều của các vùng ảnh và nó đóng vai trò quan trọng trong nâng cao chất lượng ảnh [2].

Trong [2], giá trị thuần nhất của điểm ảnh được kết nhập từ các giá trị địa phương $E_{i j}, H_{i j}, V_{i j}, R_{4, i j}$ (xem ký hiệu ở Bảng I) theo công thức sau:

$$
\begin{aligned}
H O_{i j} & =\bar{E}_{i j} \times \bar{V}_{i j} \times \bar{H}_{i j} \times \bar{R}_{4, i j} \\
& =\left(1-E_{i j}\right) \times\left(1-V_{i j}\right) \times\left(1-H_{i j}\right) \times\left(1-R_{4, i j}\right) .
\end{aligned}
$$

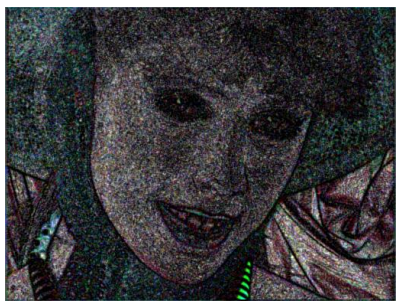

(a)

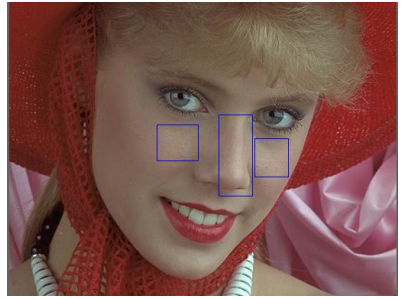

(b)
Hình 1. (a) Độ thuần nhất tính theo công thức gốc (1) [2]; (b) Ảnh nâng cao độ tương phản kênh $R, G$ và $B$ sử dụng công thức (1).

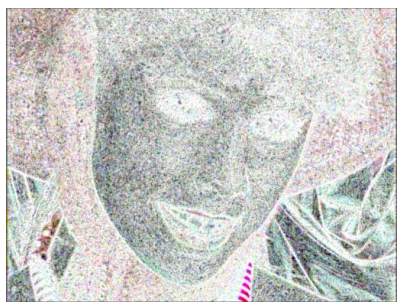

(a)

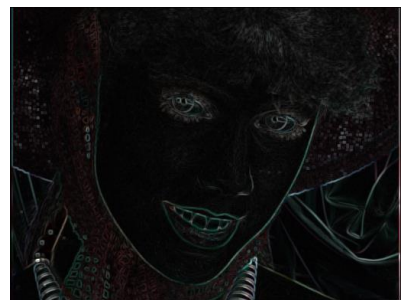

(b)
Hình 2. $\left\{H_{i j}\right\}$ (a) và $\left\{V_{i j}\right\}$ (b) 3 kênh $\mathrm{R}, \mathrm{G}$ và $\mathrm{B}$ với ảnh \#5.

Khi thử nghiệm với ảnh màu, chúng tôi nhận thấy rằng, kết hợp theo công thức (1) có thể tạo ra giá trị độ thuần nhất rất không trơn, do đó ảnh hưởng đến độ trơn của ảnh nâng cao độ tương phản đầu ra.

Hình 1(a) chứng tỏ các giá trị độ thuần nhất tại các điểm ảnh của ảnh \#5, Hình 7 và ảnh kết quả của phép nâng cao của [2] khi dùng công thức gốc (1) là không đủ trơn (Hình 1(b) ở các vùng đánh dấu ô chữ nhật).

Ở đây cần nhấn mạnh là các giá trị entropy địa phương $H_{i j}$ rất nhạy với nhiễu và sự thay đổi của giá trị mức xám, điều này đã làm cho phép kết nhập theo công thức (1) có thể không hiệu quả.

Thực tế giá trị độ thuần nhất của điểm ảnh là một giá trị mờ và chúng ta có thể áp dụng lập luận mờ để thu nhận giá trị này.

Nếu các đặc trưng địa phương được $\bar{E}_{i j}, \bar{H}_{i j}$ chuyển cho một tiếp cận tính toán với từ (computing with words) thì công thức kết nhập dạng $T_{e h}\left(\bar{E}_{i j}, \bar{H}_{i j}\right)$ cần phản ánh luật mờ như sau:

i) Nếu gradient là cao và entropy là cao, thì độ thuần nhất là cao;

ii) Nếu gradient là thấp và entropy là thấp, thì độ thuần nhất là thấp;

trong đó ký hiệu $\overline{\bullet \bullet}$ là biến ngôn ngữ với giá trị ngữ nghĩa là phủ định giá trị ngữ nghĩa của biến ngôn ngữ $(\bullet)$.

Hơn nữa nếu chúng ta bổ sung thêm các luật với từ các gia tử như "rất", "it", "vừa", v.v. với các biến ngôn ngữ như 
"homogeneity", “entropy”, "gradient”, v.v. vào tập luật mờ, các giá trị thuần nhất có thể được ước lượng bằng suy diễn của con người và vì thế sẽ mịn hơn.

Một hệ lập luật mờ nhiều điều kiện (FMCR: fuzzy multiple conditional reasoning) có dạng tổng quát như sau:

$$
\begin{aligned}
& \text { If } X_{1}=A_{11} \text { và } \ldots \text { và } X_{m}=A_{1 m} \text { then } Y=B_{1}, \\
& \text { If } X_{1}=A_{21} \text { và } \ldots \text { và } X_{m}=A_{2 m} \text { then } Y=B_{2}, \\
& \ldots \\
& \text { If } X_{1}=A_{n 1} \text { và } \ldots \text { và } X_{m}=A_{n m} \text { then } Y=B_{n},
\end{aligned}
$$

trong đó $X_{1}, X_{2}, \ldots, X_{m}$ và $Y$ là các biến ngôn ngữ, $A_{i j}, B_{i}$ $(i=1, \ldots, n ; j=1, \ldots, m)$ là các giá trị ngôn ngữ tương ứng. Ký hiệu $X_{j}=A_{i j}$ (hoặc $Y=B_{i}$ ) là một viết tắt của câu " $X_{j}$ là $A_{i}$ " (hoặc, " $Y$ là $B_{i}$ ").

Để giải hệ lập luật mờ có thể sử dụng lập luận mờ với biểu diễn tập mờ cho các từ hoặc sử dụng đại số gia tử (ĐSGT) [12]. Cách lập luận của ĐSGT khá đơn giản. Do mỗi biến ngôn ngữ $X_{i}$ và $Y$ được xem như các phần tử của các ĐSGT $A X_{i}=\left(X_{i}, C, H, \leq\right)$ và $A Y=(Y, C, H, \leq)$ tương ứng và mỗi câu của hệ luật (2) được xem như một điểm vì thế hệ luật (2) xác định một siêu mặt ngôn ngũ̃ $(m+1)$ chiều, $S_{L, m+1}$ trong không gian $X_{1} \times \cdots \times X_{m} \times Y$. Sau đó, với mỗi $X_{j}$ và $Y$, chúng ta sử dụng một ánh xạ định lượng ngữ nghĩa (SQM: Semantically Quantifying Mapping) $v_{j}$ và $v$, để gán mỗi $X_{j}$ và $Y$ với một giá trị xác định trong miền tham chiếu trong $U_{j}=[0,1]$ và $V=[0,1]$, tương ứng, $j=1,2, \ldots, m$. Vì vậy, mỗi câu của hệ luật (2) có thể biểu diễn bằng một điểm trong $[0,1]^{m} \times[0,1]$. Do đó, hệ luật (2) bây giờ đã được biểu diễn bằng siêu mặt thực $S_{R, m+1}$ của không gian $(m+1)$ chiều.

Phần còn lại của bài báo được tổ chức như sau. Mục II trình bày một số nghiên cứu liên quan đến thuật toán nâng cao độ tương phản theo hướng trực tiếp của Cheng và cộng sự. Mục III đề xuất thuật toán sử dụng phép lập luận dựa vào giá trị định lượng ngữ nghĩa của ĐSGT để ước lượng giá trị độ thuần nhất địa phương và thuật toán nâng cao độ tương phản ảnh màu. Mục IV trình bày các kết quả thực nghiệm và mục V là kết luận của bài báo.

\section{NGHIÊN CỨU LIÊN QUAN}

Để thuận tiện trong việc trình bày, trong bài báo này, chúng tôi thống nhất sử dụng các ký hiệu như đã liệt kê trong Bảng I.

\section{1. Ước lượng độ sáng nền dựa trên một độ đo thuần nhất địa phương}

Giả sử $g_{i j}$ là mức xám của một điểm ảnh $I(i, j)$ của ảnh đa cấp xám $I$ kích thước $M \times N$, và $W_{i j}$ cửa sổ lân cận tại $(i, j)$ kích thước $d \times d$. Thực hiện tuần tự các bước sau để tính giá trị thuần nhất của điểm ảnh.
Bước 1: Tính các tham số địa phương được chuẩn hóa giá trị về đoạn $[0,1]$, gradient $E_{i j}$, entropy $H_{i j}$, trung bình độ lệch chuẩn $V_{i j}$, và moment bậc $4 R_{4, i j}$ (xem Phụ lục).

Bước 2: Tính giá trị độ thuần nhất của điểm ảnh và giá trị mức xám không thuần nhất.

Tính giá trị đo độ thuần nhất tại điểm ảnh, $\beta_{i j}$, theo công thức:

$$
\beta_{i j}=\frac{H O_{i j}}{\max H O_{i j}},
$$

trong đó $H O_{i j}$ tính theo công thức (1).

Tính giá trị mức xám không thuần nhất (nonhomogeneity gray value [2]) theo công thức:

$$
\delta_{i j}=\frac{\sum_{(p, q) \in W_{i j}} g_{p q}\left(1-\beta_{p q}\right)}{\sum_{(p, q) \in W_{i j}}\left(1-\beta_{p q}\right)} .
$$

\section{Nâng cao độ tương phản dựa trên độ đo tương phản trực tiếp tại từng điểm ảnh}

Trong $[1,2]$, độ tương phản $C$ được xác định bởi

$$
C=\left|\frac{f-b}{f+b}\right|,
$$

trong đó $f$ là độ sáng của đối tượng, và $b$ là độ sáng vùng xung quanh.

Nâng cao độ tương phản dựa trên phương pháp trực tiếp, theo $[1,2]$, là việc thực hiện một dãy biến đổi $(f, b) \rightarrow$ $C_{f, b} \rightarrow C_{\text {new }} \rightarrow f_{\text {new, } f, b}$, trong đó $0 \leq C_{f, b} \leq C_{\text {new }} \leq 1$ và

$$
f_{\text {new }, f, b}= \begin{cases}b \frac{1-C_{\text {new }}}{1+C_{\text {new }}}, & f \leq b, \\ b \frac{1+C_{\text {new }}}{1-C_{\text {new }}}, & f>b .\end{cases}
$$

Cụ thể trong [2], sau khi xây dựng một độ đo thuần nhất trên kênh ảnh, phép nâng cao độ tương phản trực tiếp gồm các bước sau đây.

Bước 1: Tính giá trị mức xám không thuần nhất theo (4).

Bước 2: Tính độ tương phản của điểm ảnh $C_{i j}$ theo công thức (5) với $f=g_{i j}$ và $b=\delta_{i j}$.

Bước 3: Tính số mũ khuếch đại và biến đổi điểm ảnh (xem Phụ lục)

Một đặc tính cơ bản của thuật toán trên [2] là luật sau được thỏa mãn bởi phép nâng cao độ tương phản:

Tại từng điểm ảnh, độ thuần nhất càng cao thì múc độ nâng tương phản càng thấp. 


\section{Tổng quan về đại số gia tử}

1) Đại số gia tử của biến ngôn ngũu:

Giả sử $X$ là một biến ngôn ngữ và miền giá trị của $X$ là $\operatorname{Dom}(X)$. ĐSGT $A X$ tương ứng của $X$ là một bộ 4 thành phần $A X=(\operatorname{Dom}(X), C, H, \leq)$ trong đó $C$ là tập các phần tử sinh, $H$ là tập các gia tử và quan hệ " $\leq$ " là quan hệ cảm sinh ngữ nghĩa trên $X$ [13].

Trong ĐSGT $A X=(\operatorname{Dom}(X), C, H, \leq)$ nếu $\operatorname{Dom}(X)$ và $C$ là tập sắp thứ tự tuyến tính thì $A X$ được gọi là ĐSGT tuyến tính.

2) Các hàm đo trong ĐSGT tuyến tính:

Trong phần này, ta sử dụng ĐSGT tuyến tính $A X=$ $(X, C, H, \leq)$ với $C=\left\{c^{-}, c^{+}\right\} \cup\{0,1, W\} . H=H^{-} \cup H^{+}$, $H^{-}=\left\{h_{-1}, h_{-2}, \ldots, h_{-q}\right\}$ thỏa $h_{-1}<h_{-2}<\cdots<h_{-q}$ và $H^{+}=\left\{h_{1}, h_{2}, \ldots, h_{p}\right\}$ thỏa $h_{1}<h_{2}<\cdots<h_{p}$ và $h 0=\mathcal{I}$, với $\mathcal{I}$ là toán tử đơn vị.

Gọi $H(x)$ là tập các phần tử của $X$ sinh ra từ $x$ bởi các gia tử. Độ đo tính mờ của $x$, ta ký hiệu là $f m(x)$, là đường kính của tập $f(H(x))=\{f(u): u \in H(x)\}$.

Định nghĩa 1 ([12]): Cho ĐSGT $A X=(X, C, H, \leq)$. Hàm $f m: X \rightarrow[0,1]$ được gọi là hàm độ đo tính mờ của các phần tử trong $X$ nếu:

i) $f m\left(c^{-}\right)+f m\left(c^{+}\right)=1$ và $\sum_{h \in H} f m(h u)=f m(u)$, với $\forall u \in X$

ii) $f m(x)=0$, với mọi $x$ sao cho $H(x)=\{x\}$. Đặc biệt, $f m(0)=f m(W)=f m(1)=0$;

iii) $\forall x, y \in X, \forall h \in H, \frac{f m(h x)}{f m(x)}=\frac{f m(h y)}{f m(y)}$, tỷ lệ này không phụ thuộc vào $x, y$ và được gọi là độ đo tính mờ của gia tử $h$, ký hiệu là $\mu(h)$.

Mệnh đề 1 ([12]): Cho $f m$ là hàm độ đo tính mờ trên $X$, ta có:
i) $f m(h x)=\mu(h) f m(x), \forall x \in X$
ii) $f m\left(c^{-}\right)+f m\left(c^{+}\right)=1$
iii) $\sum_{-q \leq i \leq p, i \neq 0} f m\left(h_{i} c\right)=f m(c), \forall c \in\left\{c^{-}, c^{+}\right\}$.
iv) $\sum_{-q \leq i \leq p, i \neq 0} f m\left(h_{i} x\right)=f m(x)$
v) $\sum_{-q \leq i \leq-1} \mu\left(h_{i}\right)=\alpha$ và $\sum_{1 \leq i \leq p} \mu\left(h_{i}\right)=\beta$, trong đó $\alpha$, $\beta>0$ và $\alpha+\beta=1$.

Định nghĩa 2 ([14]): Hàm dấu sign : $X \rightarrow\{-1,0,1\}$ được định nghĩa đệ quy như sau:
i) $\operatorname{sign}\left(c^{-}\right)=-1, \operatorname{sign}\left(c^{+}\right)=+1$;
ii) $\operatorname{sign}\left(h^{\prime} h x\right)=-\operatorname{sign}(h x)$ nếu $h^{\prime} h x \neq h x$ và $h^{\prime}$ âm đối với $h$ (hoặc tương ứng với $c$, nếu $h=I \& x=c$ );
iii) $\operatorname{sign}\left(h^{\prime} h x\right)=\operatorname{sign}(h x)$, nếu $h^{\prime} h x \neq h x$ và $h^{\prime}$ dương đối với $h$ (hoặc tương ứng với $c$, nếu $h=I \& x=c$ ); iv) $\operatorname{sign}\left(h^{\prime} h x\right)=0$, nếu $h^{\prime} h x=h x$.

Mệnh đề 2 ([14]): Với bất kỳ gia tử $h \in H$ và phần tử $x \in X$, nếu $\operatorname{sign}(h x)=+1$ thì ta có $h x>x$ và nếu $\operatorname{sign}(h x)=-1$ thì $h x<x$.
Định nghĩa 3 ([12]): Cho $f m$ là hàm độ đo tính mờ trên $X$. Một hàm định lượng ngữ nghĩa $v$ trên $X$ (kết hợp với $f m$ ) được định nghĩa như sau:

$$
\text { i) } \begin{aligned}
& v(W)=\theta=f m\left(c^{-}\right)(0<\theta<1) \\
& v\left(c^{-}\right)=\theta-\alpha f m\left(c^{-}\right), \\
& v\left(c^{+}\right)=\theta-\alpha f m\left(c^{+}\right) \\
\text {ii) } & \forall j \neq 0,-q \leq j \leq p, v\left(h_{j} x\right)=v(x)+ \\
& \operatorname{sign}\left(h_{j} x\right)\left\{\sum_{i=\operatorname{sign}(j)}^{j} f m\left(h_{j} x\right)-\omega\left(h_{j} x\right) f m\left(h_{j} x\right)\right\}, \\
& \operatorname{trong} \text { đó } \omega\left(h_{j} x\right) \in\{\alpha, \beta\}, \\
& \omega\left(h_{j} x\right) \stackrel{\operatorname{def}}{=} \frac{1}{2}\left[1+\operatorname{sign}\left(h_{j} x\right) \operatorname{sign}\left(h_{p} h_{j} x\right)(\beta-\alpha)\right]
\end{aligned}
$$

Mệnh đề 3 ([12]): $\forall x \in X, 0 \leq v(x) \leq 1$.

\section{3) Phép nội suy sử dụng $S Q M$ :}

Trong ĐSGT, phương pháp giải hệ luật (2) được thực hiện như sau:

Bước 1: Xác định ĐSGT cho các biến ngôn ngữ $X_{j}$ và $Y$ tương ứng là $A X_{j}=\left(X_{j}, G_{j}, C_{j}, H_{j}, \leq_{j}\right)$ và $A Y=(Y, G$, $C, H, \leq)$.

Giả sử $v_{X_{j}}$ và $v_{Y}$ là các $\mathrm{SQM}$ của các ĐSGT $A X_{j}$ và $A Y$ của các biến ngôn ngữ $X_{j}$ và $Y$ tương ứng, $j=1,2, \ldots, m$. Gọi $S_{L}=\left\{\left\{x_{j}\right\}_{j=\overline{1, m}}, y\right\}_{i=\overline{1, n}} \subset \prod_{j=1}^{n} X_{j} \times Y$ là siêu mặt ngôn ngữ và

$S_{\text {norm }}=\left\{\left\{v_{x_{j}}\left(x_{j}\right)\right\}_{j=\overline{1, m}}, v_{Y}(y)\right\}_{x_{j} \in X_{j}, j=\overline{1, m}, y \in Y} \subset[0,1]^{m+1}$.

Hệ luật (2) sẽ được nhúng như $n$ điểm $A_{i}=\left(A_{i 1}, \ldots\right.$, $\left.A_{i m}, B_{i}\right)$ và sau đó, hệ luật (2) mô tả siêu mặt ngôn ngữ $S_{L}$ trong không gian $X_{1} \times \cdots \times X_{m} \times Y$.

Bước 2: Xác định một phương pháp nội suy trên $S_{\text {norm }}$

Tính các SQM $v_{X_{j}}\left(A_{i j}\right), v_{Y}\left(B_{j}\right)(j=\overline{1, m}, i=\overline{1, n})$ sử dụng định nghĩa 3 và các công thức (7), (8).

Siêu mặt $S_{\text {norm }} \supset\left\{\left\{v_{X_{j}}\left(A_{i j}\right)\right\}_{j=\overline{1, m}}, v_{Y}\left(B_{i}\right)\right\}_{i=\overline{1, n}}$ có thể được xác định bởi một hàm kêt nhập $m$-đối $f_{S_{\text {norm }}}, v=$ $f_{S_{\text {norm }}}\left(u_{1}, \ldots, u_{m}\right), v \in[0,1]$ và $u_{j} \in[0,1], j=\overline{1, m}$, thỏa mãn điều kiện $v_{Y}\left(B_{i}\right)=f_{S_{\text {norm }}}\left(v_{X_{1}}\left(A_{i 1}, \ldots, v_{X_{m}}\left(A_{i m}\right)\right), i=\overline{1, n}\right.$.

Chúng ta có thể sử dụng một trong rất nhiều phép nội suy đã có để thực hiện nội suy.

Bước 3: Tìm đầu ra chuẩn hóa về $[0,1] B_{0}$ tương ứng với đầu vào $A_{0}$ đã chuẩn hóa về $[0,1]$ :

$$
\begin{aligned}
A_{0} & =\left(a_{0,1}, \ldots, a_{0, m}\right), a_{0, j} \in[0,1], \text { với } j=\overline{1, m} \\
B_{0} & =f_{S_{\text {norm }}}\left(a_{0,1}, \ldots, a_{0, m}\right) \in[0,1]
\end{aligned}
$$

\section{KỸ THUÂ̂T ĐỀ XUẤT}

\section{Xây dựng độ đo thuần nhất sử dụng đại số gia tử}

Chúng tôi đã thay thế công thức kết nhập (1) bởi công thức sau:

$$
H O_{i j}=\max \left\{\bar{E}_{i j} \times \bar{H}_{i j}, \bar{V}_{i j} \times \bar{R}_{4, i j}\right\},
$$




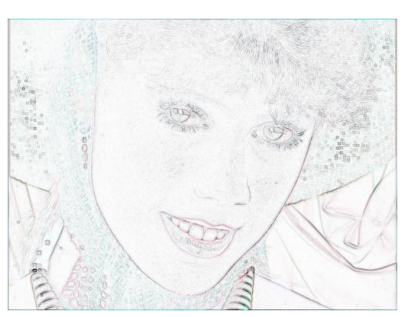

(a)

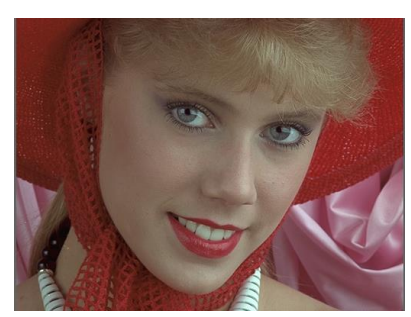

(b)
Hình 3. (a) Độ thuần nhất tính theo công thức (8); (b) Ảnh nâng cao sử dụng công thức (8).

và nhận thấy rằng công thức (8) là phù hợp cho đa dạng ảnh màu RGB (các ảnh đã được nâng cao độ tương phản khi sử dụng công thức (8) và thuật toán của [2] đều trơn).

Giá trị thuần nhất của điểm ảnh tính theo công thức (8) đã được lượng hóa đơn giản, chỉ tại những vùng mà sự trực giác thấy rõ là không thuần nhất, giá trị độ thuần nhất mới được lượng hóa có giá trị thấp.

Ngoài ra, thử nghiệm với các ảnh màu khác nhau cùng thuật toán của $[1,2]$ cho từng kênh ảnh $R, G$ và $B$ chúng tôi cũng nhận thấy cả 2 công thức (1) và công thức (8) cùng cho ảnh đầu ra của thuật toán [2] có sự tương phản mạnh ở vùng có độ thuần nhất thấp (chẳng hạn, vùng đôi mắt, xem Hình 1 (b) và $3(\mathrm{~b})$ ) và mức độ sáng của hình ảnh được nâng cao là như nhau.

Qua sự phân tích trên chúng ta có thể thấy phép kết hợp các đặc trưng địa phương thích hợp (ảnh kết quả phải trơn) nên có dạng:

$$
\begin{aligned}
H O_{i j} & =f\left(\bar{E}_{i j}, \bar{V}_{i j}, \bar{H}_{i j}, \bar{R}_{4, i j}\right) \\
& =T\left(T_{e h}\left(\bar{E}_{i j}, \bar{H}_{i j}\right), T_{h r}\left(\bar{V}_{i j}, \bar{R}_{4, i j}\right)\right) .
\end{aligned}
$$

Các đặc trưng địa phương $V_{i j}, R_{4, i j}$ biến đổi chậm nên ở đây chủ yếu là ảnh hưởng của phép kết nhập $T_{e h}\left(\bar{E}_{i j}, \bar{H}_{i j}\right)$.

Độ đo thuần nhất được xây dựng dựa trên hệ luật khá đơn giản và rõ ràng sau: Giả sử $\bar{G}(\overline{\text { gradient }}), \bar{E}$ (entropy $)$ và $T$ là các biến ngôn ngữ với miền ngữ nghĩa giá trị số được chuẩn hóa về đoạn $[0,1]$.

$\operatorname{HMR}(\bar{G}, \bar{E}, T)$ là tập luật mờ cho $\bar{G}, \bar{E}$ và $T$, gọi tắt là hệ luật HMR (Homogeneity Measure Rule), được phát biểu như sau:

R1 If $\bar{G}$ là very high và $\bar{E}$ là high Then $T$ là very high

R2 If $\bar{G}$ là very low và $\bar{E}$ là low Then $T$ là very low

R3 If $\bar{G}$ là high và $\bar{E}$ là very high Then $T$ is very high

R4 If $\bar{G}$ là low và $\bar{E}$ là very low Then $T$ là very low

R5 If $\bar{G}$ là high và $\bar{E}$ là high Then $T$ là high

R6 If $\bar{G}$ là $l o w$ và $\bar{E}$ là $l o w$ Then $T$ là $l o w$

R7 If $\bar{G}$ là little high và $\bar{E}$ là high Then $T$ là little high
Bảng II

MỐI QUAN HỆ DẤU CỦA CÁC GIA TỬ VÀ CÁC PHẦN TỬ SINH

\begin{tabular}{|c|c|c|}
\hline & V & L \\
\hline V & + & + \\
\hline L & - & - \\
\hline
\end{tabular}

R8 If $\bar{G}$ là little low và $\bar{E}$ là low Then $T$ là little low R9 If $\bar{G}$ là high và $\bar{E}$ là little high Then $T$ là little high R10 If $\bar{G}$ là low và $\bar{E}$ là little low Then $T$ là little low R11 If $\bar{G}$ là very high và $\bar{E}$ là very high Then $T$ là very very high

R12 If $\bar{G}$ là very low và $\bar{E}$ là very low Then $T$ là very very low

Tiếp theo, chúng ta xây dựng hàm $f_{S_{\text {norm }}}$ cho hệ luật HMR. Sử dụng phương pháp lập luận ĐSGT như đã trình bày trong mục II-2-3, ta có các bước để tính kết quả đầu ra khi cho biết đầu vào theo 3 bước của mục II-3-3 như sau:

Bước 1: Thiết lập các ĐSGT và các tham số tính mờ tương ứng

Ký hiệu $A G=(\bar{G}, C, w, H, \leq), A E=(\bar{E}, C, w, H, \leq)$ và $A T=(T, C, w, H, \leq), C=\left\{c^{-}, c^{+}\right\}, c^{-}=$low,$c^{+}=$high, $H=H^{-} \cup H^{+}, H^{-}=\{$little $\}, H^{+}=\{$very $\}, L \equiv$ little, $V \equiv$ very.

Đặt $\theta_{\bar{G}}=f m_{\bar{G}}\left(c^{-}\right), \alpha_{\bar{G}}=\mu_{\bar{G}}(L), \theta_{\bar{E}}=f m_{\bar{E}}\left(c^{-}\right), \alpha_{\bar{E}}=$ $\mu_{\bar{E}}(L), \theta_{T}=f m_{T}\left(c^{-}\right), \alpha_{T}=\mu_{T}(L)$, trong đó $\theta_{\bar{G}}, \alpha_{\bar{G}}, \theta_{\bar{E}}$, $\alpha_{\bar{E}}, \theta_{T}, \alpha_{T} \in(0,1)$.

Mối quan hệ dấu của các gia tử được xác định như trong Bảng II. Từ Bảng này ta có $\operatorname{sign}\left(V c^{-}\right)=\operatorname{sign}\left(V I c^{-}\right)=$ $\left.\operatorname{sign}(V I) \times \operatorname{sign}\left(I c^{-}\right)=1 \times \operatorname{sign}\left(I c^{-}\right)=-1\right)$.

\section{Bước 2:}

2.1. Tính các $S Q M$ của các thành phần bên trái và bên phải hệ luật mờ sử dụng Bảng III, trong đó $U$ đóng vai trò của $\bar{G}, \bar{E}$ và $T$.

2.2. Với một toán tử "và" 2 ngôi $\mathrm{AND}:[0,1]^{2} \rightarrow[0,1]$, $\operatorname{AND}(\bar{G}, \bar{E})=\bar{G} \times \bar{E}$, chúng ta có mảng các điểm nội suy bề mặt $S_{\text {norm }}$ (ở đây $m=2$ ) của hệ luật HMR trong Bảng IV.

Từ đây có thể sử dụng phép nội suy đơn giản $f_{S_{\text {norm }}}$ như phép tuyến tính từng đoạn trên các mốc nội suy.

\section{Nhận xét:}

Cho trước cặp giá trị ngữ nghĩa đầu vào $(\bar{G}, \bar{E}) \in[0,1]^{2}$, ta xác định giá trị ngữ nghĩa đầu $\operatorname{ra} h_{T} \in[0,1]$ như sau: $h_{T}=f_{S_{\text {norm }}}(\operatorname{AND}(\bar{G}, \bar{E}))$;

Hàm $f_{S_{\text {norm }}}$ cho hệ luật HMR được xây dựng như trên được ký hiệu là $T_{\mathrm{HA}}$, AND hay gọn hơn là $T_{\mathrm{HA}}$ khi đã cho trước toán tử AND. 
Bảng III

BẢNG GIÁ TRI ĐỒ ĐO TÍNH MỜ VÀ SQM TƯƠNG ỨNG VỚI AG, AE, AT

\begin{tabular}{|c|c|c|c|}
\hline Tham số & Với $\bar{G}$ & Với $\bar{E}$ & Với $T$ \\
\hline$\mu_{U}\left(c^{+}\right)$ & $\bar{\theta}_{\bar{G}}=1-\theta_{\bar{G}}$ & $\bar{\theta}_{\bar{E}}=1-\theta_{\bar{E}}$ & $\bar{\theta}_{T}=1-\theta_{T}$ \\
\hline$\mu_{U}(V)$ & $\beta_{\bar{G}}=1-\alpha_{\bar{G}}$ & $\beta_{\bar{E}}=1-\alpha_{\bar{E}}$ & $\beta_{T}=1-\alpha_{T}$ \\
\hline$v_{U}($ low $)$ & $\beta_{\bar{G}} \theta_{\bar{G}}$ & $\beta_{\bar{E}} \theta_{\bar{E}}$ & $\beta_{T} \theta_{T}$ \\
\hline $\begin{array}{l}v_{U}(\text { V.low }) \\
(\text { very low })\end{array}$ & $\beta_{\bar{G}}^{2} \theta_{\bar{G}}$ & $\beta_{\bar{E}}^{2} \theta_{\bar{E}}$ & $\beta_{T}^{2} \theta_{T}$ \\
\hline $\begin{array}{l}v_{U}(\text { L.low) } \\
\text { (little low) }\end{array}$ & $\begin{array}{l}\nu_{\bar{G}}(\text { low })+ \\
\alpha_{\bar{G}} \beta_{\bar{G}} \theta_{\bar{G}}\end{array}$ & $\begin{array}{l}v_{\bar{E}}(l o w)+ \\
\alpha_{\bar{E}} \beta_{\bar{E}} \theta_{\bar{E}}\end{array}$ & $\begin{array}{l}v_{T}(\text { low })+ \\
\alpha_{T} \beta_{T} \theta_{T}\end{array}$ \\
\hline$v_{U}(h i g h)$ & $1-\beta \bar{G} \bar{\theta} \bar{G}$ & $1-\alpha_{\bar{E}} \bar{\theta}_{\bar{E}}$ & $1-\alpha_{T} \bar{\theta}_{T}$ \\
\hline $\begin{array}{l}v_{U}(V . h i g h) \\
\text { very high }\end{array}$ & $1-\beta_{\bar{G}}^{2} \bar{\theta}_{\bar{G}}$ & $1-\beta_{\bar{E}}^{2} \bar{\theta}_{\bar{E}}$ & $1-\beta_{T}^{2} \bar{\theta}_{T}$ \\
\hline $\begin{array}{l}v_{U}(\text { L.high }) \\
(\text { little high })\end{array}$ & $\begin{array}{l}\nu_{\bar{G}}(h i g h) \\
-\alpha_{\bar{G}} \beta_{\bar{G}} \bar{\theta}_{\bar{G}}\end{array}$ & $\begin{array}{l}v_{\bar{E}}(h i g h) \\
-\alpha_{\bar{E}} \beta \overline{\bar{E}} \overline{\theta_{\bar{E}}}\end{array}$ & $\begin{array}{l}\nu_{T}(h i g h) \\
-\alpha_{T} \beta_{T} \bar{\theta}_{T}\end{array}$ \\
\hline $\begin{array}{l}\nu_{U}(V . V . l o w) \\
\text { (very very low) }\end{array}$ & $\beta_{\bar{G}}^{3} \theta_{\bar{G}}$ & $\beta_{\bar{E}}^{3} \theta_{\bar{E}}$ & $\beta_{T}^{3} \theta_{T}$ \\
\hline $\begin{array}{l}v_{U}(\text { V.V.high }) \\
(\text { very very high })\end{array}$ & $1-\beta_{\bar{G}}^{3} \bar{\theta}_{\bar{G}}$ & $1-\beta \frac{3}{E} \bar{\theta} \bar{E}$ & $1-\beta_{T}^{3} \bar{\theta}_{T}$ \\
\hline
\end{tabular}

Bảng IV

BẢNG GIÁ TRI CÁC MỐC NÔI SUY DƯA TRÊN TOÁN TƯ AND CỦA HỆ LUẬT HMR

\begin{tabular}{|c|c|}
\hline Chỉ số luật & Các điểm nội suy $(x, y) \in[0,1]^{2} x \rightarrow y$ \\
\hline R1 & $\operatorname{AND}\left(\nu_{\bar{G}}(V . h i g h), v_{\bar{E}}(h i g h)\right) \rightarrow v_{T}(V . h i g h)$ \\
\hline R2 & $\operatorname{AND}\left(v_{\bar{G}}(V . l o w), v_{\bar{E}}(V\right.$. low $\left.)\right) \rightarrow v_{T}(V . l o w)$ \\
\hline R3 & $\operatorname{AND}\left(v_{\bar{G}}(V . h i g h), v_{\bar{E}}(V . h i g h)\right) \rightarrow v_{T}(V . h i g h)$ \\
\hline R4 & $\operatorname{AND}\left(v_{\bar{G}}(\right.$ low $\left.), v_{\bar{E}}(V . l o w)\right) \rightarrow v_{T}(V . l o w)$ \\
\hline R5 & $\operatorname{AND}\left(v_{\bar{G}}(h i g h), v_{\bar{E}}(h i g h)\right) \rightarrow v_{T}(h i g h)$ \\
\hline R6 & $\operatorname{AND}\left(v_{\bar{G}}(\right.$ low $), v_{\bar{E}}($ low $\left.)\right) \rightarrow v_{T}($ low $)$ \\
\hline R7 & $\operatorname{AND}\left(v_{\bar{G}}(\right.$ L.high $\left.), v_{\bar{E}}(h i g h)\right) \rightarrow v_{T}($ L.high $)$ \\
\hline R8 & $\operatorname{AND}\left(v_{\bar{G}}(\right.$ L.low $), v_{\bar{E}}($ low $\left.)\right) \rightarrow v_{T}($ L.low $)$ \\
\hline R9 & $\operatorname{AND}\left(v_{\bar{G}}(h i g h), v_{\bar{E}}(L . h i g h)\right) \rightarrow v_{T}($ L.high $)$ \\
\hline R10 & $\operatorname{AND}\left(v_{\bar{G}}(\right.$ low $), v_{\bar{E}}($ L.low $\left.)\right) \rightarrow v_{T}($ L.low $)$ \\
\hline R11 & $\operatorname{AND}\left(v_{\bar{G}}(V . h i g h), v_{\bar{E}}(V . h i g h)\right) \rightarrow v_{T}(V . h i g h)$ \\
\hline R12 & $\operatorname{AND}\left(v_{\bar{G}}(V . l o w), v_{\bar{E}}(V . l o w)\right) \rightarrow v_{T}(V . V . l o w)$ \\
\hline
\end{tabular}

Mệnh đề 4: Hàm $T_{\mathrm{HA}}:[0,1]^{2} \rightarrow[0,1]$ bảo toàn thứ tự, nghĩa là, với mọi $a, b, a^{\prime}, b^{\prime} \in[0,1], a \leq a^{\prime}, b \leq b^{\prime}$, ta có $T_{\mathrm{HA}}(a, b) \leq T_{\mathrm{HA}}\left(a^{\prime}, b^{\prime}\right)$.

Chứng minh: Do

i) AND bảo toàn thứ tự,
Thuật toán 1: Xác định mức độ thuần nhất tại mỗi điểm ảnh HA-HMR

Đầu vào: Ảnh đa cấp xám $I$, có kích thước $M \times N$.

Tham số: $\theta_{g}, \alpha_{g r}, \theta_{e p}, \alpha_{e p}, \theta_{h o}, \alpha_{h o} \in(0,1)$ của ĐSGT $\mathrm{AGr}, \mathrm{AEp}$ và $\mathrm{AHo}$

Đầu ra: Bảng giá trị độ thuần nhất tại mỗi điểm ảnh.

Bước 1: Tính gradient, entropy, độ lệch chuẩn và mô men bậc 4 và chuẩn hóa về $[0,1]$ các giá trị này.

for mỗi điểm ảnh $g_{i j}$

1.1. Tính $E_{i j}, H_{i j}, V_{i j}, R_{4, i j}$ dùng công thức (A.1)

đến (A.4) trong phụ lục (xem [11])

1.2. Tính $\bar{E}_{i j}=1-E_{i j}, \bar{H}_{i j}=1-H_{i j}, \bar{V}_{i j}=1-V_{i j}$,

$\bar{R}_{4, i j}=1-R_{4, i j}$

end for

Bước 2: Với toán tử $\mathrm{AND}$, sử dụng Bảng III và Bảng IV xây dựng hàm $T_{H A}$ cho hệ luật HMR(AGr, AEp, AHo) của các biến ngôn ngữ $\mathrm{Gr}$ (gradient), Ep (entropy) và Ho (homogeneity) với các tham số độ đo mờ $\theta_{g}, \alpha_{g r}, \theta_{e p}$, $\alpha_{e p}, \theta_{h o}, \alpha_{h o} \in(0,1)$.

Bước 3: Tính độ thuần nhất tại từng điểm ảnh

3.1. Tính $E H_{i j}$ và $H O_{i j}$

for mỗi điểm ảnh $g_{i j}$

Tính $E H_{i j}=T_{\mathrm{HA}}\left(\bar{E}_{i j}, \bar{H}_{i j}\right)$

Tính $H O_{i j}=\min \left\{E H_{i j}, \bar{V}_{i j} \times \bar{R}_{4, i j}\right\}$

end for

3.2. Chuẩn hóa

for mỗi điểm ảnh $g_{i j}$

Tính $\beta_{i j}=\frac{H O_{i j}}{\max \left\{H O_{i j}\right\}}$

end for

Trả về $\left\{\beta_{i j}\right\}$

ii) Các $\mathrm{SQM} v_{\bar{G}}, v_{\bar{E}}$ và $v_{T}$ bảo toàn thứ tự trên $\bar{G}, \bar{E}$ và $T$ (xem định nghĩa 2 và định nghĩa 3 ),

suy ra $T_{\mathrm{HA}}$ bảo toàn thứ tự tại các mốc nội suy (xem Bảng IV).

Do $f_{S_{\text {norm }}}$ được tạo thành từ phép nội suy tuyến tính từng đoạn giữa các mốc nội suy, nên bảo toàn thứ tự $T_{\mathrm{HA}}$ trên $[0,1]^{2}$. Sử dụng $T_{\mathrm{HA}}$, chúng ta xác định một độ đo thuần nhất của điểm ảnh như sau:

$$
H O_{i j}=T\left(T_{\mathrm{HA}}\left(\bar{E}_{i j}, \bar{H}_{i j}\right), \bar{V}_{i j}, \bar{R}_{4, i j}\right) .
$$

Kỹ thuật xây dựng độ đo thuần nhất sử dụng ĐSGT được thực hiện theo Thuật toán 1 . Thuật toán này có độ phức tạp $O(M \times N)$.

Hình 4 minh họa kết quả của xây dựng độ đo thuần nhất sử dụng ĐSGT. 


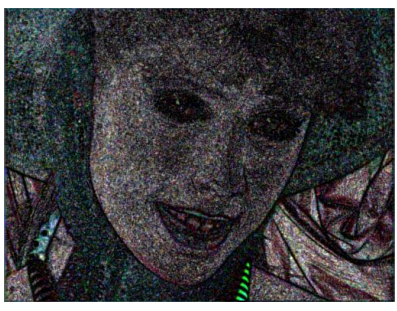

(a)

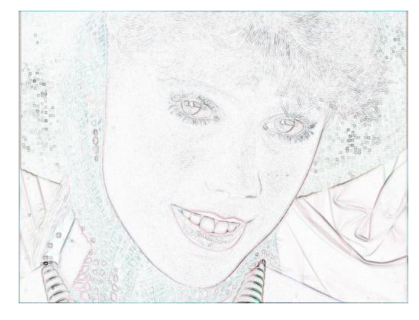

(b)
Hình 4. (a) Các giá trị thuần nhất của kênh $\mathrm{V}$ của ảnh \#5 sử dụng [11]; (b) Giá trị thuần nhất sử dụng ĐSGT.

\section{Nâng cao độ tương phản của ảnh màu với độ đo thuần nhất dề xuất}

Trước tiên, đề xuất xây dựng một biến đổi ảnh $\mathrm{F}$ của từng kênh ảnh xám của tổ hợp kênh ảnh đầu vào. Khi đó độ tương phản được tính theo công thức sau:

$$
C_{i j}=\left|\frac{F\left(g_{i j}\right)-\delta_{i j}(F)}{F\left(g_{i j}\right)+\delta_{i j}(F)}\right| \text {. }
$$

Để xây dựng một biến đổi ảnh phù hợp với nhiều loại ảnh khác nhau như ảnh tối, ảnh sáng, ảnh có độ tương phản thấp và ảnh có độ tương phản cao, chúng tôi đề xuất sử dụng phân cụm mờ C-Mean (FCM: Fuzzy C-mean [15]) để ước lượng dải động của mức xám của từng kênh ảnh của ảnh đa kênh. Trong [13] đã trình bày một cách xác định dải động của miền giá trị mức xám bằng cách sử dụng thuật toán phân cụm mờ FCM, khi đó các cụm ảnh có tính chất đều hơn, do đó việc xác định dải động mức xám là tương đối dễ dàng.

Sau khi phân cụm, việc ước lượng dải động mức xám của từng cụm sẽ dễ dàng hơn do tính đồng nhất cao của giá trị mức xám trong một cụm.

Với một tổ hợp $K$ kênh ảnh của ảnh $I$ (trong một biểu diễn màu), để thuận tiện chúng ta ký hiệu $I_{\overline{1, K}}=$ $\left\{I_{1}, I_{2}, \ldots, I_{K}\right\}$, sử dụng thuật toán $\mathrm{FCM}$ phân cụm $I_{\overline{1, K}}$ thành $C$ cụm, $C \geq 2$. Thuật toán lặp FCM cực tiểu hóa hàm mục tiêu:

$$
J(V, \mu)=\min _{V, \mu} \sum_{i, j} \sum_{c=1}^{C} \mu_{i, j, c}^{2}\left\|I_{\overline{1, K}}(i, j)-V_{c}\right\|^{2},
$$

với độ đo khoảng cách Euclid

$$
\left\|I_{\overline{1, K}}(i, j)-V_{c}\right\|=\sqrt{\sum_{k=1}^{K}\left(I_{k}(i, j)-V_{c}(k)\right)^{2}}
$$

và các ràng buộc biến như sau:

i) $\mu_{i, j, c} \in[0,1], 1 \leq c \leq C$;

ii) $\sum_{c=1}^{C} \mu_{i, j, c}=1$, với mọi $1 \leq i \leq M$ và $1 \leq j \leq N$;

iii) $\sum_{i, j} \mu_{i, j, c}>0$, với mọi $1 \leq c \leq C$.
Như vậy, với FCM chúng ta nhận được bảng các giá trị độ thuộc từng cụm cho từng điểm ảnh là $\left\{\mu_{i, j, c}\right\}$, trong đó $1 \leq c \leq C, 1 \leq I \leq M$ và $1 \leq j \leq N$.

Histogram mờ theo từng kênh $I_{k}, 1 \leq k \leq K$, ký hiệu là $h_{c}^{k}$ được xác định như sau:

$$
\begin{aligned}
& h_{c}^{k}(g)=\sum_{(i, j) \in\left\{g_{i j}=\left(g_{i j}^{1}, g_{i j}^{2}, \ldots, g_{i j}^{K}\right): g_{i j}^{k}=g\right\}} \mu_{i, j, c}, \\
& g=L_{k, \min }, \ldots, L_{k, \max } .
\end{aligned}
$$

Ký hiệu $\operatorname{His}_{I_{k}}(g)$ là histogram của kênh ảnh $k$, ta có mệnh đề sau.

Mệnh đề 5: Với mọi $k=\overline{1, K}$ và với mọi $L_{k, \min } \leq g \leq$ $L_{k, \max }$, ta có:

$$
\sum_{1 \leq c \leq C} h_{c}^{k}(g)=\operatorname{His}_{I_{k}}(g) .
$$

Chứng minh:

$$
\begin{aligned}
\sum_{1 \leq c \leq C} h_{c}^{k}(g) & \left.=\sum_{c=1}^{C} \sum_{(i, j) \in\left\{g_{i j}=\left(g_{i j}^{1}, g_{i j}^{2}, \ldots, g_{i j}^{K}\right): g_{i j}^{k}=g\right\}} \mu_{i, j, c}\right) \\
= & \sum_{(i, j) \in\left\{g_{i j}=\left(g_{i j}^{1}, g_{i j}^{2}, \ldots, g_{i j}^{K}\right): g_{i j}^{k}=g\right\}} \sum_{c=1}^{C} \mu_{i, j, c} \\
= & \sum_{(i, j) \in\left\{g_{i j}=\left(g_{i j}^{1}, g_{i j}^{2}, \ldots, g_{i j}^{K}\right): g_{i j}^{k}=g\right\}} 1 \\
= & \operatorname{His}_{I_{k}}(g) .
\end{aligned}
$$

Dải động mức xám của cụm $c$ là $\left[B_{k, 1, c}, B_{k, 2, c}\right]$ trong kênh $k$ được ước lượng như sau:

$$
\begin{aligned}
& B_{k, 1, c}=\underset{B \in\left[L_{k, \min }, L_{k, \max }\right]}{\operatorname{argmin}}\left\{\sum_{g=L_{k, \min }}^{B} h_{c}^{k}(g)>f_{\text {cut }} \sum_{g=L_{k, \min }}^{L_{k, \max }} h_{c}^{k}(g)\right\} \\
& B_{k, 2, c}=\underset{B \in\left[L_{k, \min }, L_{k, \max }\right]}{\operatorname{argmin}}\left\{\sum_{B}^{L_{k, \max }} h_{c}^{k}(g)<f_{\text {cut }} \sum_{g=L_{k, \min }}^{L_{k, \max }} h_{c}^{k}(g)\right\}
\end{aligned}
$$

Hình 5 minh họa một histogram mờ của cụm 4 chiếu trên kênh $\mathrm{R}$ sau khi phân cụm ảnh RGB đầu vào thành 5 cụm bằng thuật toán FCM.

Với mỗi $k=\overline{1, K}$, chúng ta xác định một biến đổi $F_{k}$ cho kênh ảnh $I_{k}$ như sau:

$$
\begin{aligned}
& F_{k}(i, j)=\left[L_{k, \min }+\right. \\
& \left.\quad \frac{L_{k, \max }-L_{k, \min }}{C} \sum_{c=1}^{C} S_{\mathrm{f}}\left(I_{k}(i, j), B_{k, 1, c}, V_{k, c}, B_{k, 2, c}\right)\right]
\end{aligned}
$$


Thuật toán 2: Nâng cao độ tương phản của ảnh màu HSV và độ đo thuần nhất HA-HMR

\section{Đầu vào:}

Ảnh màu $I$ trong biểu diễn màu RGB,

kích thước $M \times N$.

Tham số $C \in N^{+}$, ngưỡng $f_{\text {cut }}\left(f_{\text {cut }}>0\right.$, đủ nhỏ), $d$ $(d \times d$ là kích thước cửa sổ $)$.

Đầu ra:

Ảnh màu RGB $I_{\text {new }}$, và tùy chọn trả về:

Giá trị tương phản trung bình $\mathrm{CM}_{\mathrm{R}}, \mathrm{CM}_{\mathrm{G}}, \mathrm{CM}_{\mathrm{B}}$

Giá trị $E_{\text {avg }}, H_{\text {avg }}$

Bước 1: Gọi $\left(I_{\mathrm{H}}, I_{\mathrm{S}}, I_{\mathrm{V}}\right)$ là biểu diễn màu của $I$ trong không gian màu HSV. Lượng hóa để coi các kênh $I_{\mathrm{S}}, I_{\mathrm{V}}$ như là các ảnh đa cấp xám.

Bước 2: Với dữ liệu đầu vào là tổ hợp kênh $\left(I_{\mathrm{S}}, I_{\mathrm{V}}\right)$, tham số số cụm là $C$ và ngưỡng $f_{\text {cut }}$, thực hiện phân cụm FCM để ước lượng $C$ dải động mức xám $\left[B_{k, 1, c}, B_{k, 2, c}\right]$ với $k \in\{\mathrm{S}, \mathrm{V}\}$ (xem công thức (14)).

Bước 3: Xác định ảnh biến đổi $F_{\mathrm{S}}, F_{\mathrm{V}}$ của kênh $I_{\mathrm{S}}, I_{\mathrm{V}}$ tương ứng theo công thức (15).

\section{Bước 4:}

4.1. Tính các giá trị thuần nhất của $F_{\mathrm{S}}, F_{\mathrm{V}}$ dựa trên ĐSGT sử dụng HA-HMR.

4.2. Tính tham số của [2] cho kênh $F_{\mathrm{S}}, F_{\mathrm{V}}$ như đã trình bày trong phụ lục với kích thước cửa sổ $d \times d$, cụ thể là các giá trị mức xám không thuần nhất $\left\{\delta_{\mathrm{S}, i j}\right\},\left\{\delta_{\mathrm{V}, i j}\right\}$, số mũ khuếch đại $\left\{\xi_{\mathrm{S}, i j}\right\},\left\{\xi_{\mathrm{V}, i j}\right\}$ tại từng điểm ảnh của kênh $F_{\mathrm{S}}$ và kênh $F_{\mathrm{V}}$.

Bước 5: Tính độ tương phản và xác định kênh ảnh xám mới của kênh $F_{\mathrm{S}}$ và kênh $F_{\mathrm{V}}, F_{\mathrm{S}} \rightarrow I_{\mathrm{S} \text {, new }}, F_{\mathrm{V}} \rightarrow I_{\mathrm{V} \text {,new }}$ như sau:

Với kênh $F_{\mathrm{S}} \equiv F_{\mathrm{S}}\left(I_{\mathrm{S}}\right)$ và kênh $F_{\mathrm{V}} \equiv F_{\mathrm{V}}\left(I_{\mathrm{V}}\right)$ :

Tính độ tương phản

$$
\begin{aligned}
& C_{\mathrm{S}, i j}=\left|\frac{F_{\mathrm{S}}\left(g_{S, i j}\right)-\delta_{i j}\left(F_{\mathrm{S}}\right)}{F_{\mathrm{S}}\left(g_{S, i j}\right)+\delta_{i j}\left(F_{\mathrm{S}}\right)}\right|, \\
& C_{\mathrm{V}, i j}=\left|\frac{F_{\mathrm{V}}\left(g_{V, i j}\right)-\delta_{i j}\left(F_{\mathrm{V}}\right)}{F_{\mathrm{V}}\left(g_{V, i j}\right)+\delta_{i j}\left(F_{\mathrm{V}}\right)}\right|
\end{aligned}
$$

trong đó $k=\overline{1, K}, i=\overline{1, M}, j=\overline{1, N},[x]$ chỉ phần nguyên của số thực $x$ và hàm $S_{\mathrm{f}}$ đã được định nghĩa trong [3].

Sử dụng độ đo thuần nhất HA-HMR được xây dựng trong Thuật toán 1 , kỹ thuật nâng cao độ tương phản của ảnh màu trong biểu diễn màu HSV được thực hiện theo Thuật toán 2.

Không tính đến thuật toán FCM và phép nội suy giải hệ lập luận mờ HMR của ĐSGT, Thuật toán 2 có độ phức tạp tương đương thuật toán gốc [2]. Mô hình kiến trúc hệ thống đề xuất được xây dựng như Hình 6 .
Tính giá trị mức xám mới của kênh $\mathrm{S}$ và $\mathrm{V}$

$$
\begin{aligned}
& I_{\mathrm{S}, \text { new }}(i, j)=\left\{\begin{array}{c}
\delta_{\mathrm{S}, i j} \frac{1-C_{\mathrm{S}, i j}^{\xi_{\mathrm{S}, i j}^{t}}}{\epsilon_{\mathrm{S}, i j}^{t}}, \quad g_{\mathrm{S}, i j} \leq \delta_{\mathrm{S}, i j} \\
\delta_{\mathrm{S}, i j} \frac{1+C_{\mathrm{S}, i j}^{\epsilon_{\mathrm{S}, i j}}}{1-\epsilon_{\mathrm{S}, i j}^{t}, i j}, \quad g_{\mathrm{S}, i j}>\delta_{\mathrm{S}, i j}
\end{array}\right.
\end{aligned}
$$

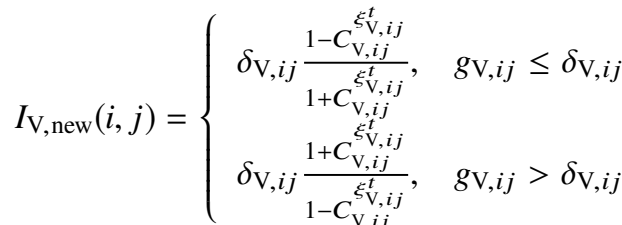

Lưu ý, ở đây kênh $\mathrm{S}$ được đánh chỉ số $k=1$, kênh $\mathrm{V}$ được đánh chỉ số $k=2$.

Bước 6: Chuyển ảnh $\left(I_{\mathrm{H}}, I_{\mathrm{S}, \text { new }}, I_{\mathrm{V} \text {,new }}\right)$ trong biểu diễn màu HSV về biểu diễn màu RGB, ta được ảnh $I_{\text {new }}$.

Bước 7: Bước tùy chọn, tính các chỉ số khách quan $\mathrm{CM}\{\mathrm{R}, \mathrm{G}, \mathrm{B}\}, E_{\text {avg và }} H_{\text {avg }}$

7.1. Tính tham số của [2] cho kênh $I_{\mathrm{R}}, I_{\mathrm{G}}$ và $I_{\mathrm{B}}$ của ảnh gốc $I$ (xem phụ lục) với kích thước cửa sổ $d \times d$, cụ thể là các giá trị mức xám không thuần nhất $\left\{\delta_{\mathrm{R}, i j}\right\},\left\{\delta_{\mathrm{G}, i j}\right\}$, và $\left\{\delta_{\mathrm{B}, i j}\right\}$ của kênh $I_{\mathrm{R}}, I_{\mathrm{G}}$ và $I_{\mathrm{B}}$ tương ứng.

7.2. Tính $\mathrm{CM}_{\mathrm{R}}, \mathrm{CM}_{\mathrm{G}}, \mathrm{CM}_{\mathrm{B}}$ theo công thức (18)

$$
\begin{aligned}
\mathrm{CM}_{\mathrm{R}}= & \frac{\sum_{i j} \frac{\left|I_{\text {new }, \mathrm{R}}(i, j)-\delta_{\mathrm{R}, i j}\right|}{I_{\text {new }, \mathrm{R}}(i, j)+\delta_{\mathrm{R}, i j}}}{M \times N} \\
\mathrm{CM}_{\mathrm{G}}=\frac{\sum_{i j} \frac{\left|I_{\text {new }, \mathrm{G}}(i, j)-\delta_{\mathrm{G}, i j}\right|}{I_{\text {new }, \mathrm{G}}(i, j)+\delta_{\mathrm{G}, i j}}}{M \times N} & \sum_{i j} \frac{\left|I_{\text {new }, \mathrm{B}}(i, j)-\delta_{\mathrm{B}, i j}\right|}{I_{\text {new }, \mathrm{B}}(i, j)+\delta_{\mathrm{B}, i j}} \\
\mathrm{CM}_{\mathrm{B}}= & \frac{M \times N}{M \times N}
\end{aligned}
$$

7.3. Tính $E_{\text {avg }}=E_{\text {avg }}\left\{I_{\text {new }, R}, I_{\text {new }, G}, I_{\text {new }, B}\right\}$,

$H_{\text {avg }}=H_{\text {avg }}\left\{I_{\text {new }, R}, I_{\text {new }, G}, I_{\text {new }, B}\right\}$ theo (17) và (20).

Trả về: $I_{\text {new }}$, và các tùy chọn được trả về

$\mathrm{CM}_{R}, \mathrm{CM}_{G}, \mathrm{CM}_{B}, E_{\text {avg }}, H_{\text {avg }}$.

\section{THỰC NGHIỆM}

Trong thực nghiệm, chúng tôi đã sử dụng đa dạng các kiểu ảnh màu, các kênh màu của ảnh vệ tinh. Các ảnh đa màu được chọn điển hình từ loại ảnh tối, ảnh sáng, ảnh có độ tương phản các kênh là thấp, ảnh có độ tương phản các kênh là cao, v.v. Tập ảnh màu (đánh số \#1, \#2,...,\#6) được dùng để thể hiện trong khuôn khổ bài báo này thu nhận từ tập ảnh RGB được công bố trong [16] (ảnh từ \#3 đến \#5 trong Hình 7), ảnh vệ tinh huyện Lạc Thủy của Việt Nam (ảnh \#6 trong Hình 7). 


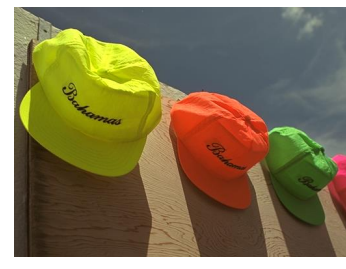

(a)

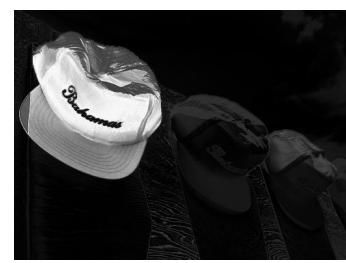

(c)

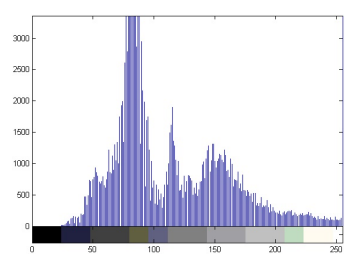

(b)

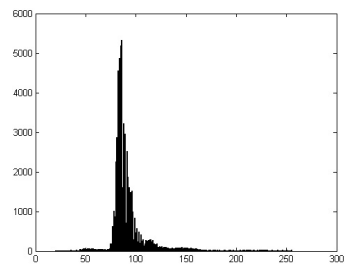

(d)
Hình 5. (b) Histogram kênh R; (c) Giá trị độ thuộc $\left\{\mu_{4, i j}\right\}$-cụm số 4/5; (d) histogram mờ kênh $\mathrm{R}$, cụm số 4/5.

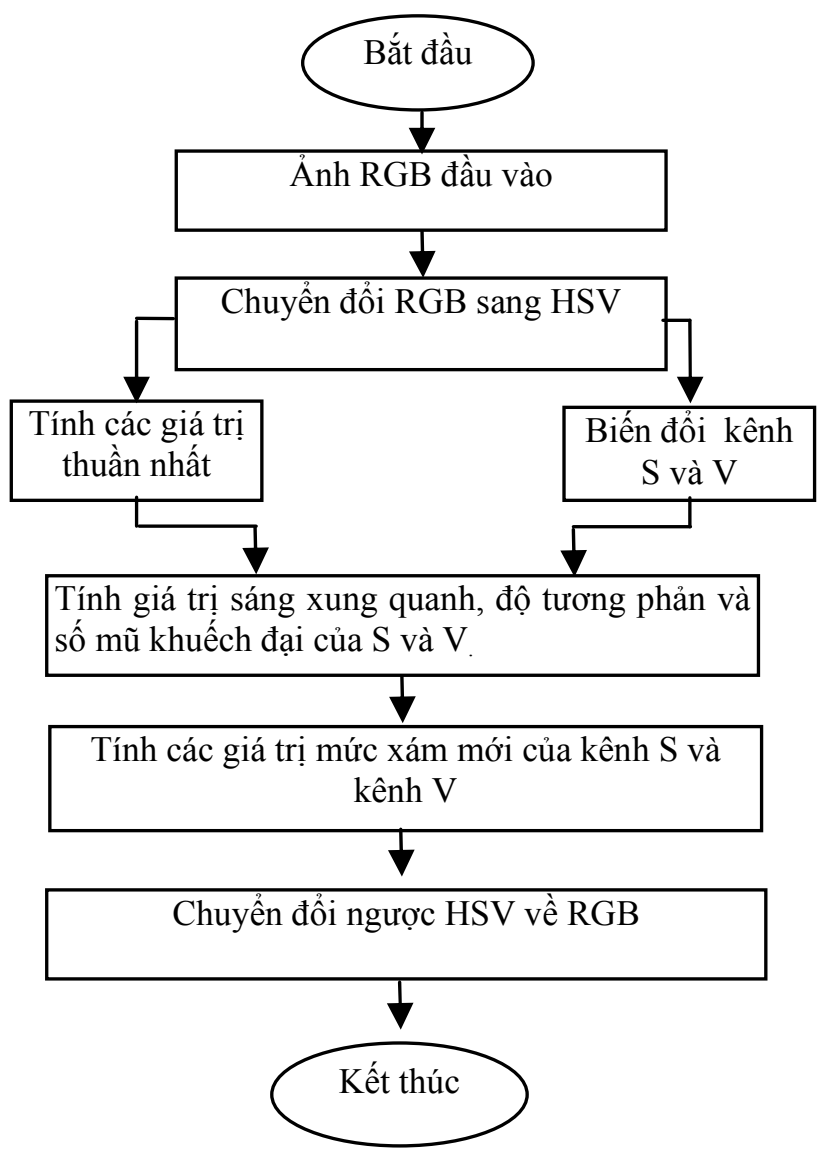

Hình 6. Lưu đồ xử lý của các thuật toán đề xuất.

\section{Tính độ thuần nhất kênh ảnh}

Các giá trị tham số ĐSGT của các biến ngôn ngữ Gr, Ep, Ho thiết kế độ đo thuần nhất như Bảng $\mathrm{V}$, trong đó $U$ đóng vào của các biến ngôn ngữ Gr, Ep và Ho.

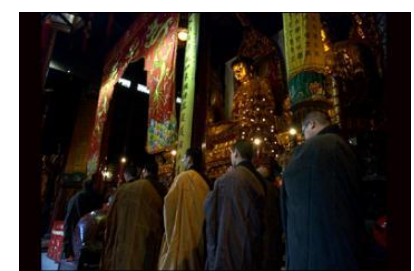

\#1: Kích thước $352 \times 254$

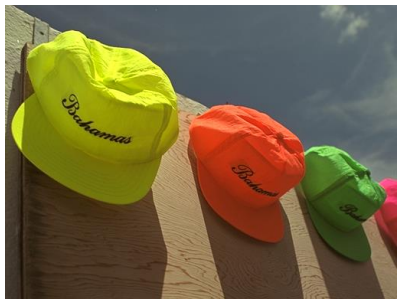

\#3: Kích thước $512 \times 384$

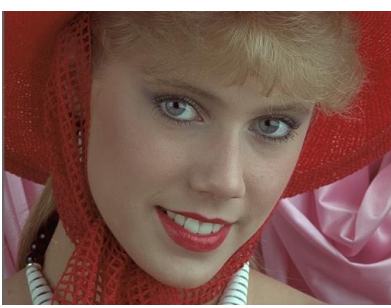

\#5: Kích thước $512 \times 384$

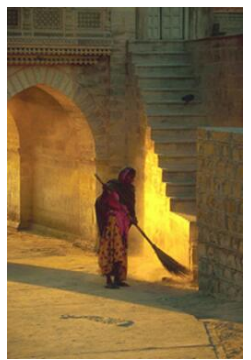

\#1: Kích thước $352 \times 254$

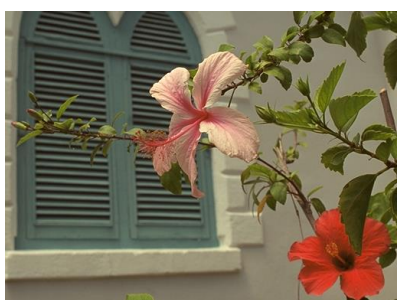

\#4: Kích thước $512 \times 384$

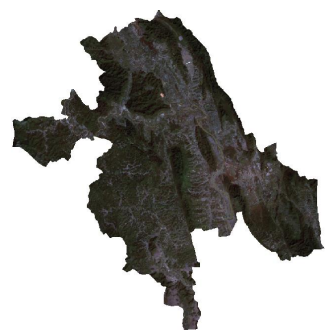

\#6: Kích thước $633 \times 647$
Hình 7. Một số ảnh gốc được dùng cho thử nghiệm.

Bảng $\mathrm{V}$

CÁC THAM SỐ CƠ SỞ CỦA ĐSGT AGR, AEP VÀ AHO

\begin{tabular}{|l|c|c|c|}
\hline Tham số & $\begin{array}{c}\text { Với Gr } \\
\text { gradient }\end{array}$ & $\begin{array}{c}\text { Với Ep } \\
\text { entropy }\end{array}$ & $\frac{\text { Với Ho }}{\text { homogeneity }}$ \\
\hline$\mu_{U}\left(c^{-1}\right)$ & 0,5 & 0,5 & 0,5 \\
\hline$\mu_{U}($ little $)$ & 0,5 & 0,5 & 0,5 \\
\hline
\end{tabular}

\section{2. Đánh giá độ đo HA-HMR}

Khi so sánh độ đo đề xuất với độ đo gốc được công bố trong [2], chúng tôi sẽ sử dụng các chỉ số khách quan để đánh giá.

Các chỉ số được dùng trong bài báo này cụ thể là:

1) Chỉ số độ tương phản trực tiếp của một kênh ảnh $I_{k^{\prime}}$ so với một kênh ảnh gốc $I_{k}\left(I_{k^{\prime}}\right.$ và $I_{k}$ có cùng kích thước $M \times N)$, được cho như sau:

$$
\operatorname{CM}\left(I_{k}, I_{k}^{\prime}\right)=\frac{\sum_{i j} \frac{\left|I_{k}^{\prime}(i, j)-\delta_{k, i j}\right|}{I_{k}^{\prime}(i, j)+\delta_{k, i j}}}{M \times N},
$$

trong đó $\delta_{k, i j}$ là giá trị mức xám không thuần nhất tại điểm ảnh $(i, j)$ của $I_{k}$ (xem ký hiệu ở Bảng I và [2]). 
Bảng VI

CÁC PHÉP KẾT HợP GIÁ TRỊ ĐỊA PHƯƠNG KHÁC NHAU

\begin{tabular}{|c|c|}
\hline$H O_{i j}=T\left(\bar{E}_{i j}, \bar{H}_{i j}, \bar{V}_{i j}, \bar{R}_{4, i j}\right)$ & Công thức \\
\hline$H O_{1}$ & $H O_{i j}=\max \left\{\bar{E}_{i j} \times \bar{H}_{i j}, \bar{V}_{i j} \times \bar{R}_{4, i j}\right\}$ \\
\hline $\mathrm{HO}_{2}$ & $H O_{i j}=\max \left(T_{\text {Aczel }}\left(\bar{E}_{i j}, \bar{H}_{i j}\right), \bar{V}_{i j} \times \bar{R}_{4, i j}\right)$ \\
\hline (Aczel) & $T_{\text {Aczel }}(a, b)=e^{-\left((-\log (a))^{2}+(-\log (b))^{2}\right)^{0.5}}$ \\
\hline $\begin{array}{l}\mathrm{HO}_{3} \\
\text { (Dombi) }\end{array}$ & $\begin{array}{l}H O_{i j}=\max \left(T_{\text {Dombi }}\left(\bar{E}_{i j}, \bar{H}_{i j}\right), \bar{V}_{i j} \times \bar{R}_{4, i j}\right) \\
T_{\text {Dombi }}(a, b)=\frac{1}{1+\left(\left(\frac{1-a}{a}\right)^{2}+\left(\frac{1-b}{b}\right)^{2}\right)^{0.5}}\end{array}$ \\
\hline $\begin{array}{l}\mathrm{HO}_{4} \\
\text { (Frank) }\end{array}$ & $\begin{array}{l}H O_{i j}=\max \left(T_{\text {Frank }}\left(\bar{E}_{i j}, \bar{H}_{i j}\right), \bar{V}_{i j} \times \bar{R}_{4, i j}\right) \\
T_{\text {Frank }}(a, b)=\log \left(1+\frac{\left(e^{a}-1\right)\left(e^{b}-1\right)}{e-1}\right)\end{array}$ \\
\hline $\begin{array}{l}\mathrm{HO}_{5} \\
\text { (Hamacher) }\end{array}$ & $\begin{array}{l}H O_{i j}=\max \left(T_{\text {Hamacher }}\left(\bar{E}_{i j}, \bar{H}_{i j}\right), \bar{V}_{i j} \times \bar{R}_{4, i j}\right) \\
T_{\text {Hamacher }}(a, b)=\frac{a b}{2 a b+1-a-b}\end{array}$ \\
\hline $\begin{array}{l}\mathrm{HO}_{6} \\
\text { (Yager) }\end{array}$ & $\begin{array}{l}H O_{i j}=\max \left(T_{\text {Yager }}\left(\bar{E}_{i j}, \bar{H}_{i j}\right), \bar{V}_{i j} \times \bar{R}_{4, i j}\right) \\
T_{\text {Yager }}(a, b)=\frac{(a b)^{0.5}}{(a b)^{0.5}+((1-a)(1-b))^{0.5}}\end{array}$ \\
\hline HA-HMR & $H O_{i j}=\max \left(T_{\mathrm{HA}}\left(\bar{E}_{i j}, \bar{H}_{i j}\right), \bar{V}_{i j} \times \bar{R}_{4, i j}\right)$ \\
\hline
\end{tabular}

2) Chỉ số entropy được cho như sau:

$$
\begin{aligned}
E\left(I_{k}\right) & =\sum_{g=L \min _{k}}^{L \max _{k}} p_{k}(g) \log _{2}\left(p_{k}(g)\right), \\
E_{\text {avg }}\left(I_{\overline{1, K}}\right) & =\frac{\sum_{k=1}^{K} E\left(I_{k}\right)}{K},
\end{aligned}
$$

trong đó $p_{k}(g) \stackrel{\text { def }}{=} \frac{\#\left\{I_{k}(i, j)=g\right\}}{M \times N}$ và quy ước $0 \cdot \log _{2}(0)=0$. Giá trị của chỉ số entropy cao thì có thể xem ảnh là giàu tính chi tiết.

3) Chỉ số fuzzy-entropy được cho như sau:

Giả sử $g_{k} \in\left[L_{k, \min }, L_{k, \max }\right] \rightarrow \mu\left(g_{k}\right) \in[0,1]$ là một phép mờ hóa nào đó,

$$
\begin{aligned}
& H_{\mu}\left(I_{k}\right)=\sum_{g=L_{k, \text { min }}}^{L_{k, \max }}\left(\mu(g) \log _{2}(\mu(g))\right. \\
&\left.+(1-\mu(g)) \log _{2}(1-\mu(g))\right) .
\end{aligned}
$$

Dưới đây chúng ta sẽ dùng phép mờ hóa tự nhiên:

$$
g \rightarrow \mu(g) \stackrel{\text { def }}{=} \frac{g-L_{k, \text { min }}}{L_{k, \text { max }}-L_{k, \text { min }}} .
$$

Chúng ta viết gọn $H$ thay cho $H_{\mu}$, và định nghĩa

$$
H_{\text {avg }}\left(I_{1, K}\right)=\frac{\sum_{k=1}^{K} H\left(I_{k}\right)}{K} .
$$

Với các ảnh thử nghiệm bước đầu tiên là tính các giá trị mức xám không thuần nhất $\left\{\delta_{R, i j}\right\},\left\{\delta_{G, i j}\right\}$, và $\left\{\delta_{B, i j}\right\}$ của 3 kênh $R, G$ và $B$ tương ứng. Các giá trị này được
Bảng VII

GIÁ TRI CHỈ SỔ ẢNH THỂ HIÊ̂N

\begin{tabular}{|l|l|l|l|l|l|}
\hline Ảnh & $\mathrm{CM}_{\mathrm{R}}$ & $\mathrm{CM}_{\mathrm{G}}$ & $\mathrm{CM}_{\mathrm{B}}$ & $E_{\text {avg }}$ & $H_{\text {avg }}$ \\
\hline$\# 1$ & 0,1180 & 0,1914 & 0,2482 & 5,9395 & 0,3456 \\
\hline$\# 2$ & 0,0160 & 0,0191 & 0,0314 & 7,3150 & 0,8216 \\
\hline$\# 3$ & 0,0154 & 0,0188 & 0,0548 & 7,4847 & 0,8001 \\
\hline$\# 4$ & 0,0256 & 0,0298 & 0,0511 & 7,4536 & 0,8642 \\
\hline$\# 5$ & 0,0170 & 0,0293 & 0,0345 & 7,3092 & 0,8504 \\
\hline$\# 6$ & 0,0273 & 0,0304 & 0,0364 & 3,4443 & 0,2861 \\
\hline
\end{tabular}

dùng để tính độ đo tương phản $\mathrm{CM}$ trên từng kênh $\mathrm{R}$, $G$ và $B$ của ảnh đầu vào và ảnh kết quả.

Bảng VII là giá trị chỉ số của các ảnh \#1-\#6, được tính từ các công thức (16) đến (20).

\section{Các kết quả và luận giải}

Trong phần này chúng tôi trình bày thử nghiệm nâng cao độ tương phản sử dụng biến đổi ảnh trên hai kênh ảnh $\mathrm{S}$ và $\mathrm{V}$ trong biểu diễn màu HSV. Các bước thử nghiệm được thực hiện như sau:

○ Đầu tiên, chuyển biểu diễn màu RGB sang biểu diễn màu HSV của ảnh đầu vào. Phân cụm dữ liệu mức xám tổ hợp kênh $\mathrm{S}$ và kênh $\mathrm{V}$ với tham số được chọn như trong thử nghiệm $A$ (số cụm $C=5$ ) trên từng kênh ảnh $\mathrm{S}$ và kênh $\mathrm{V}$ riêng rẽ ước lượng $\left\{B_{1, c, k}, B_{2, c, k}\right\}$ $(k \in\{\mathrm{S}, \mathrm{V}\})$ và thực hiện biến đổi ảnh cho kênh $\mathrm{S}$ và 
Bảng VIII

GIÁ TRI CHỈ SỐ CMR $\mathrm{CMO}_{\mathrm{R}}$ TỪNG ẢNH VÀ CÁC PHƯƠNG PHÁP KẾT HỢP ĐỂ TẠO GIÁ TRI THUẦN NHẤT

\begin{tabular}{|c|c|c|c|c|c|c|}
\hline Độ do/Ảnh & $\# 1$ & $\# 2$ & $\# 3$ & $\# 4$ & $\# 5$ & $\# 6$ \\
\hline$H O_{1}$ & 0,3692 & 0,1806 & 0,1688 & 0,2002 & 0,2132 & 0,1494 \\
\hline$H O_{2}$ & 0,3687 & 0,1805 & 0,1687 & 0,1993 & 0,2144 & 0,1494 \\
\hline$H O_{3}$ & 0,3687 & 0,1805 & 0,1687 & 0,1993 & 0,2144 & 0,1450 \\
\hline$H O_{4}$ & 0,3688 & 0,1805 & 0,1687 & 0,1992 & 0,2144 & 0,1493 \\
\hline$H O_{5}$ & 0,3687 & 0,1805 & 0,1686 & 0,1993 & 0,2144 & 0,1451 \\
\hline$H O_{6}$ & 0,3691 & 0,1806 & 0,1687 & 0,1997 & 0,2160 & 0,1454 \\
\hline HA-HMR & $\mathbf{0 , 3 8 8 9}$ & $\mathbf{0 , 1 8 1 3}$ & $\mathbf{0 , 1 8 0 2}$ & $\mathbf{0 , 2 0 6 5}$ & $\mathbf{0 , 2 1 6 1}$ & $\mathbf{0 , 1 5 0 5}$ \\
\hline
\end{tabular}

Bảng IX

GIÁ TRI CHỈ SỐ $\mathrm{CM}_{\mathrm{G}}$ CHO TỪNG ẢNH VÀ CÁC PHƯƠNG PHÁP KẾT HỢP ĐỂ TẠO GIÁ TRI THUẦN NHẤT

\begin{tabular}{|c|c|c|c|c|c|c|}
\hline Độ do/Ảnh & $\# 1$ & $\# 2$ & $\# 3$ & $\# 4$ & $\# 5$ & $\# 6$ \\
\hline$H O_{1}$ & 0,3780 & 0,1815 & 0,1703 & 0,2016 & 0,2142 & 0,1503 \\
\hline$H O_{2}$ & 0,3773 & 0,1814 & 0,1701 & 0,2007 & 0,2154 & 0,1503 \\
\hline$H O_{3}$ & 0,3773 & 0,1814 & 0,1701 & 0,2007 & 0,2154 & 0,1458 \\
\hline$H O_{4}$ & 0,3774 & 0,1814 & 0,1701 & 0,2006 & 0,2154 & 0,1502 \\
\hline$H O_{5}$ & 0,3774 & 0,1814 & 0,1701 & 0,2006 & 0,2154 & 0,1459 \\
\hline$H O_{6}$ & 0,3778 & 0,1814 & 0,1702 & 0,2011 & 0,2168 & 0,1463 \\
\hline HA-HMR & $\mathbf{0 , 4 0 0 0}$ & $\mathbf{0 , 1 8 2 2}$ & $\mathbf{0 , 1 8 1 4}$ & $\mathbf{0 , 2 0 8 0}$ & $\mathbf{0 , 2 1 7 0}$ & $\mathbf{0 , 1 6 2 3}$ \\
\hline
\end{tabular}

kênh $\mathrm{V}$ tương ứng sử dụng hàm biến đổi $F_{\mathrm{S}}, F_{\mathrm{V}}$ (công thức (18)).

- Thứ hai, xác định giá trị độ tương phản theo Thuật toán 2 và các bước liên quan cho kênh ảnh $\mathrm{S}$ và $\mathrm{V}$ đã biến đổi ở bước thứ nhất với kích thước cửa sổ $3 \times 3$ và tham số $t=0,25$ (công thức (A.8)).

- Thứ ba, tổng hợp lại các kênh ảnh $\mathrm{H}$ gốc, kênh $\mathrm{S}$ và kênh $\mathrm{V}$ đã nâng cao độ tương phản, sau đó biến đổi ngược từ biểu diễn HSV sang biểu diễn RGB.

○ Cuối cùng, tính giá trị mức xám không thuần nhất tại từng điểm ảnh ứng với các kênh ảnh $R, G$ và $B$ (các giá trị này được dùng để tính độ đo tương phản trung bình trên từng kênh $\mathrm{R}, \mathrm{G}$ và $\mathrm{B})$.

Giá trị trung bình độ tương phản trực tiếp $\mathrm{CM}$ trên kênh $R, G$ và $B$ trong biểu diễn màu $R G B$ ảnh đầu vào được tính với ảnh giá trị mức xám trung bình không thuần nhất của từng kênh $\mathrm{R}, \mathrm{G}$ và $\mathrm{B}$ của ảnh $\mathrm{RGB}$ gốc và ảnh kết quả đầu ra khi sử dụng Thuật toán 2 với các $H O_{k}, k=1, \ldots, 6$ và phương pháp đề xuất (HA-HMR) thể hiện ở các Bảng VIII đến Bảng XII.

Bảng VIII đến Bảng XII của kết quả thực nghiệm của các ảnh \#1-\#6 đã thể hiện các chỉ số khách quan độ tương phản trực tiếp trên từng kênh $\mathrm{R}, \mathrm{G}$ và $\mathrm{B}$ khi sử dụng phép kết nhập 4 đặc trưng địa phương $\mathrm{HO}_{7}$ đều cho kết quả cao hơn khi sử dụng các $H O_{k}, k=1, \ldots, 6$. Chỉ số khách quan
Bảng $X$

GIÁ TRI CHỈ SỐ CM $\mathrm{B}_{\mathrm{B}}$ CHO TỪNG ẢNH VÀ CÁC PHƯƠNG PHÁP KẾT HỢP ĐỂ TẠO GIÁ TRI THUẦN NHẤT

\begin{tabular}{|c|c|c|c|c|c|c|}
\hline Độ do/Ånh & $\# 1$ & $\# 2$ & $\# 3$ & $\# 4$ & $\# 5$ & $\# 6$ \\
\hline$H O_{1}$ & 0,4200 & 0,1851 & 0,1869 & 0,2126 & 0,2148 & 0,1498 \\
\hline$H O_{2}$ & 0,4192 & 0,1851 & 0,1868 & 0,2118 & 0,2160 & 0,1498 \\
\hline$H O_{3}$ & 0,4192 & 0,1851 & 0,1868 & 0,2118 & 0,2160 & 0,1451 \\
\hline$H O_{4}$ & 0,4194 & 0,1850 & 0,1869 & 0,2117 & 0,2160 & 0,1497 \\
\hline$H O_{5}$ & 0,4193 & 0,1851 & 0,1868 & 0,2118 & 0,2160 & 0,1453 \\
\hline$H O_{6}$ & 0,4197 & 0,1851 & 0,1869 & 0,2121 & 0,2176 & 0,1457 \\
\hline HA-HMR & $\mathbf{0 , 4 4 1 8}$ & $\mathbf{0 , 1 8 6 0}$ & $\mathbf{0 , 2 0 4 8}$ & $\mathbf{0 , 2 1 9 4}$ & $\mathbf{0 , 2 1 7 7}$ & $\mathbf{0 , 1 6 2 6}$ \\
\hline
\end{tabular}

Bảng XI

GIÁ TRI CHỈ SỐ $E_{a v g}$ CHO TỪNG ẢNH VÀ CÁC PHƯƠNG PHÁP KẾT HợP ĐỂ TẠO GIÁ TRI THUẦN NHẤT

\begin{tabular}{|c|c|c|c|c|c|c|}
\hline Độ đo/Ảnh & $\# 1$ & $\# 2$ & $\# 3$ & $\# 4$ & $\# 5$ & $\# 6$ \\
\hline$H O_{1}$ & 6,1126 & 7,2722 & 7,3154 & 7,5641 & 7,3993 & 4,2105 \\
\hline$H O_{2}$ & 6,1129 & 7,2712 & 7,3127 & 7,5456 & 7,4098 & 4,2174 \\
\hline$H O_{3}$ & 6,1123 & 7,2698 & 7,3122 & 7,5464 & 7,4076 & 4,2051 \\
\hline$H O_{4}$ & 6,1175 & 7,2684 & 7,3144 & 7,5462 & 7,4167 & 4,2228 \\
\hline$H O_{5}$ & 6,1101 & 7,2758 & 7,3096 & 7,5631 & 7,4168 & 4,2165 \\
\hline$H O_{6}$ & 6,1094 & 6,8158 & 6,9599 & 7,3155 & 7,2337 & 4,2239 \\
\hline HA-HMR & $\mathbf{6 , 1 5 0 9}$ & $\mathbf{7 , 3 8 6 2}$ & $\mathbf{7 , 5 1 2 4}$ & $\mathbf{7 , 6 3 0 7}$ & $\mathbf{7 , 5 8 3 3}$ & $\mathbf{4 , 3 6 7 5}$ \\
\hline
\end{tabular}

Bảng XII

GIÁ TRỊ CHỈ SỐ $H_{a v g}$ CHO TỪNG ẢNH VÀ CÁC PHƯƠNG PHÁP KẾT HỢP ĐỂ TẠO GIÁ TRI THUẦN NHẤT

\begin{tabular}{|c|c|c|c|c|c|c|}
\hline Độ đo/Ånh & $\# 1$ & $\# 2$ & $\# 3$ & $\# 4$ & $\# 5$ & $\# 6$ \\
\hline$H O_{1}$ & 0,3999 & $\mathbf{0 , 6 9 4 3}$ & 0,7198 & 0,7640 & $\mathbf{0 , 7 8 9 1}$ & 0,4599 \\
\hline$H O_{2}$ & 0,4022 & 0,6944 & 0,7201 & 0,7648 & 0,7909 & 0,4606 \\
\hline$H O_{3}$ & 0,4021 & 0,6944 & 0,7201 & 0,7648 & 0,7909 & 0,4424 \\
\hline$H O_{4}$ & 0,4023 & 0,6944 & 0,7201 & 0,7648 & 0,7909 & 0,4612 \\
\hline$H O_{5}$ & 0,4020 & 0,6944 & 0,7201 & 0,7645 & 0,7909 & 0,4420 \\
\hline$H O_{6}$ & 0,4017 & 0,6944 & 0,7200 & 0,7646 & 0,7904 & $\mathbf{0 , 4 4 1 4}$ \\
\hline HA-HMR & $\mathbf{0 , 3 9 8 4}$ & $\mathbf{0 , 6 9 4 3}$ & $\mathbf{0 , 7 1 9 7}$ & $\mathbf{0 , 7 6 1 0}$ & 0,7907 & 0,4501 \\
\hline
\end{tabular}

$E_{\text {avg }}$ khi áp dụng thuật cũng cao hơn với HA-HMR. Cũng

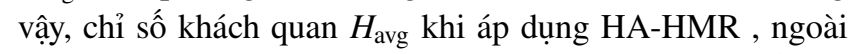
ảnh \#5 và ảnh \#6, đều có giá trị nhỏ hơn so với chỉ số ảnh kết quả khi áp dụng $H O_{k}, k=1, \ldots, 6$.

\section{KẾT LUÂ̂N}

Trong bài báo này chúng tôi đã đề xuất hai đóng góp mới cho phương pháp nâng cao độ tương phản của ảnh màu theo tiếp cận trực tiếp. Thứ nhất, chúng tôi đề xuất một độ đo thuần nhất mới của điểm ảnh khi sử dụng lập luận mờ với thuật toán dựa trên ĐSGT để kết nhập các giá trị địa phương (tại từng điểm ảnh) như gradient, entropy, 


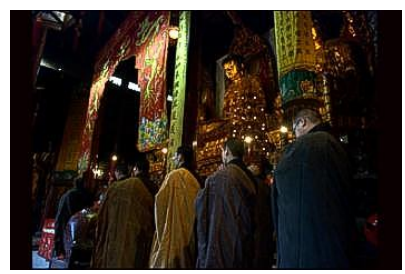

(a)

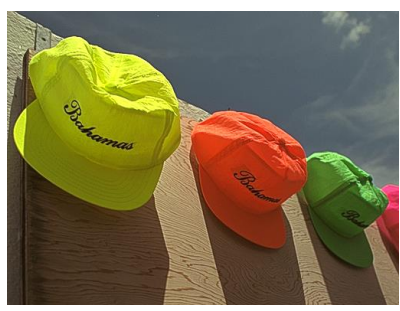

(e)

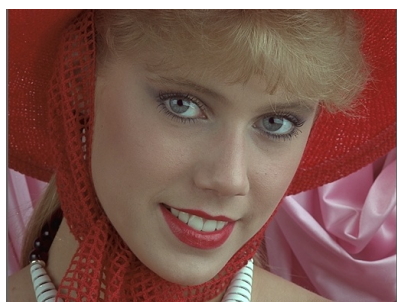

(1)

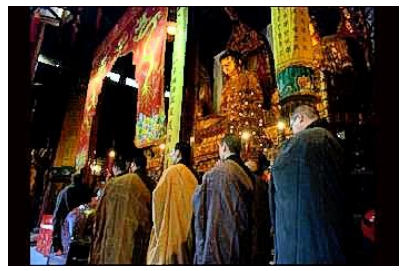

(b)

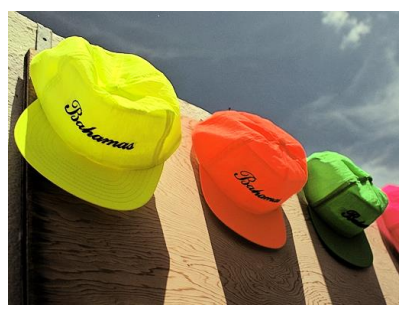

(g)

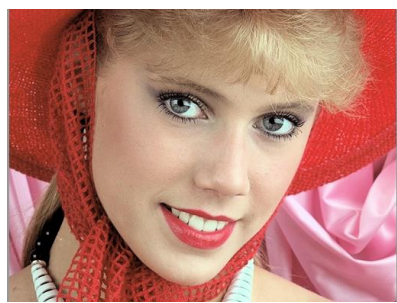

(m)

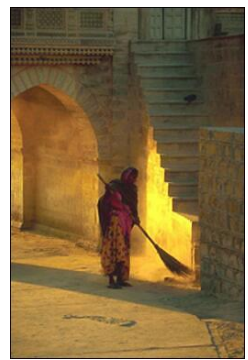

(c)

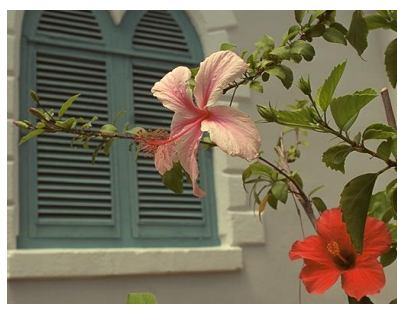

(h)

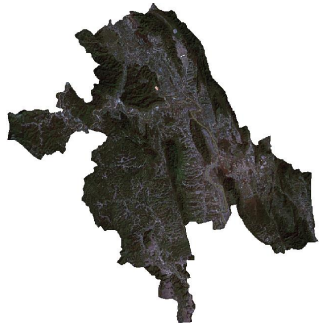

(n)

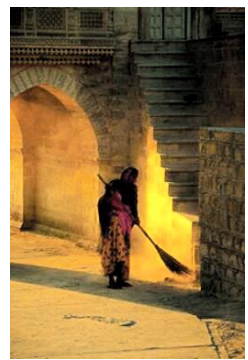

(d)

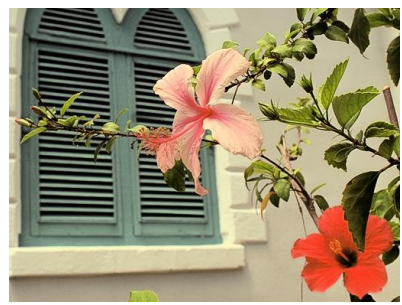

(k)

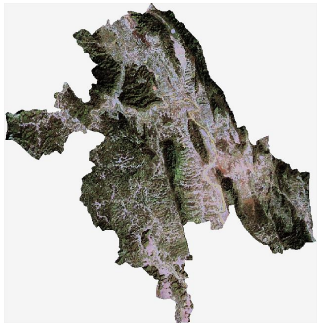

(p)

Hình 8 . Thử nghiệm cho ảnh \#1, \#2... và \#6. Ảnh kết quả (các ảnh phía bên trái ) khi sử dụng [11] Ảnh kết quả (các ảnh phía bên phải) sử dụng Thuật toán 2 với $\mathrm{HO}_{7}$ đề xuất.

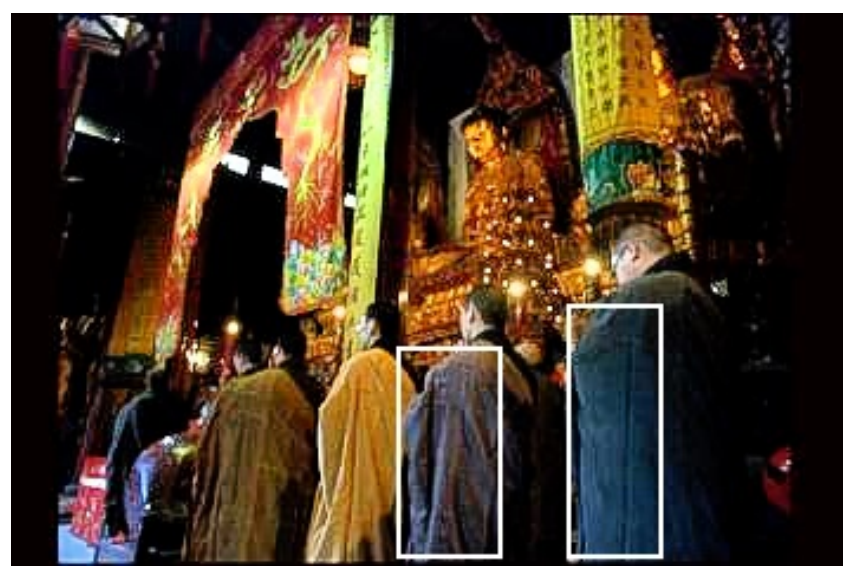

Hình 9. Ảnh kết quả sử dụng Thuật toán 2 với phép kết nhập gốc [11] $H O_{i j}=\bar{E}_{i j} \times \bar{H}_{i j} \times \bar{V}_{i j} \times \bar{R}_{4, i j}$ cho ảnh \#1 và ảnh kết quả không trơn.

độ lệch chuẩn trung bình và moment bậc 4 so với cách kết nhập của [2]. Thứ hai, chúng tôi cũng đề xuất một thuật toán mới theo phương pháp nâng cao độ tương phản trực tiếp cho ảnh màu trong biểu diễn màu HSV dựa trên độ đo thuần nhất mới đã được xây dựng bằng ĐSGT và phép biến đổi ảnh. Kết quả thực nghiệm đã chứng tỏ rằng các kỹ thuật đã đề xuất của chúng tôi đã làm việc tốt với đa dạng ảnh màu. Độ đo thuần nhất đề xuất hoạt động hiệu quả hơn các độ đo sử dụng các toán tử kết nhập mờ $T$-norm thông dụng. Ảnh nâng cao độ tương phản cũng trơn, tăng được độ sáng toàn bộ ảnh.

\section{LờI CẢM ƠN}

Nghiên cứu này được Viện Hàn lâm Khoa học và Công nghệ Việt Nam tài trợ theo chương trình VAST01.05/15-16.

\section{PHỤ LỤC}

Tính các tham số địa phương được chuẩn hóa giá trị về đoạn $[0,1]$, gradient $E_{i j}$, entropy $H_{i j}$, trung bình độ lệch chuẩn $V_{i j}$, và moment bậc $4 R_{4, i j}$ :

Tính cường độ biên ảnh

$e=\left\{e_{i j}\right\}$ là giá trị cường độ biên ảnh xám đầu vào bằng một toán tử xác định ảnh biên như toán tử Sobel. 
Giá trị trung bình, độ lệch chuẩn

$\mu=\left\{\mu_{i j}\right\}, v=\left\{v_{i j}\right\}$, trong đó $\mu_{i j}$ là giá trị trung bình mức xám, $v_{i j}$ là độ lệch chuẩn mức xám lấy tại lân cận điểm ảnh $g_{i j}$

$$
\mu_{i j}=\frac{\sum_{(p, q) \in W_{i j}} g_{p q}}{d^{2}}, v_{i j}=\sqrt{\frac{\sum_{(p, q) \in W_{i j}}\left(g_{p q}-\mu_{i j}\right)^{2}}{d^{2}}}
$$

Tính giá trị entropy địa phương

$$
h_{i j}=\frac{\sum_{k=1}^{L} p_{k} \log p_{k}}{2 \log d}, p_{k}=\frac{\#\left\{(p, q) \in W_{i j}: g_{p q}=k\right\}}{d^{2}}
$$

Tính moment bậc 4

$$
\gamma_{4, i j}=\frac{\sum_{(p, q) \in W_{i j}}\left(g_{p q}-\mu_{i j}\right)^{4}}{d^{2}-1}
$$

Chuẩn hóa về miền giá trị là đoạn $[0,1]$

$$
\begin{aligned}
& E_{i j}=\frac{e_{i j}}{\max \left\{e_{i j}\right\}} ; \quad V_{i j}=\frac{v_{i j}}{\max \left\{v_{i j}\right\}} \\
& H_{i j}=\frac{h_{i j}}{\max \left\{h_{i j}\right\}} ; \quad R_{4, i j}=\frac{\gamma_{4, i j}}{\max \left\{\gamma_{4, i j}\right\}}
\end{aligned}
$$

Tính giá trị độ tương phản tại từng điểm ảnh và số mũ khuếch đại

Giá trị độ tương phản

$$
C_{i j}=\left|\frac{g_{i j}-\delta_{i j}}{g_{i j}+\delta_{i j}}\right|
$$

Số mũ khuếch đại

$$
\zeta_{i j}=\zeta_{\min }+\frac{\left(\zeta_{\max }-\zeta_{\min }\right)\left(\beta_{i j}-\beta_{\min }\right)}{\beta_{\max }-\beta_{\min }},
$$

trong đó

$$
\zeta_{\min }=\frac{g_{k}-g_{1}}{g_{\max }-g_{1}}
$$

$\zeta_{\max }=1, g_{k}, g_{1}$ là các đỉnh của histogram được xác định theo [10]

Nâng độ tương phản

$$
C_{i j}^{\prime}=C_{i j}^{\zeta_{i j}^{t}}
$$

trong đó $t \in\{0.25,0.5\}$ là tham số [11]

Tính giá trị mức xám mới tại từng điểm ảnh

$$
g_{i j}^{\prime}=\left\{\begin{array}{cl}
\delta_{i j} \frac{1-C_{i j}^{\prime}}{1+C_{i j}^{\prime}}=\delta_{i j} \frac{1-C_{i j}^{\zeta_{i j}^{t}}}{1+C_{i j}^{\zeta_{i j}^{t}}}, & g_{i j} \leq \delta_{i j} \\
\delta_{i j} \frac{1+C_{i j}^{\prime}}{1-C_{i j}^{\prime}}=\delta_{i j} \frac{1+C_{i j}^{\zeta_{i j}^{t}}}{1-C_{i j}^{\zeta_{i j}^{t}}}, & g_{i j}>\delta_{i j}
\end{array}\right.
$$

\section{TÀI LIÊU THAM KHẢO}

[1] H.-D. Cheng and H. Xu, "A novel fuzzy logic approach to contrast enhancement," Pattern Recognition, vol. 33, no. 5, pp. 809-819, 2000.

[2] H. Cheng, M. Xue, and X. Shi, "Contrast enhancement based on a novel homogeneity measurement," Pattern Recognition, vol. 36, no. 11, pp. 2687-2697, 2003.

[3] S. S. Agaian, B. Silver, and K. A. Panetta, "Transform coefficient histogram-based image enhancement algorithms using contrast entropy," IEEE Transactions on Image Processing, vol. 16, no. 3, pp. 741-758, 2007.

[4] T. Arici, S. Dikbas, and Y. Altunbasak, "A histogram modification framework and its application for image contrast enhancement," IEEE Transactions on Image Processing, vol. 18, no. 9, pp. 1921-1935, 2009.

[5] A.-O. Boudraa and E.-H. S. Diop, "Image contrast enhancement based on 2D Teager-Kaiser operator," in IEEE International Conference on Image Processing (ICIP 2008). IEEE, 2008, pp. 3180-3183.

[6] M. Hanmandlu and D. Jha, "An optimal fuzzy system for color image enhancement," IEEE Transactions on Image Processing, vol. 15, no. 10, pp. 2956-2966, 2006.

[7] S. Lee, "An efficient content-based image enhancement in the compressed domain using retinex theory," IEEE Transactions on Circuits and Systems for Video Technology, vol. 17, no. 2, pp. 199-213, 2007.

[8] J. Soha and A. Schwartz, "Multispectral histogram normalization contrast enhancement," in $5^{\text {th }}$ Canadian Symposium on Remote Sensing, 1979, pp. 86-93.

[9] A. P. Dhawan, G. Buelloni, and R. Gordon, "Enhancement of mammographic features by optimal adaptive neighborhood image processing," IEEE Transactions on Medical Imaging, vol. 5, no. 1 , pp. 8-15, 1986.

[10] A. Beghdadi and A. Le Negrate, "Contrast enhancement technique based on local detection of edges," Computer Vision, Graphics, and Image Processing, vol. 46, no. 2, pp. 162-174, 1989.

[11] L. Dash and B. N. Chatterji, "Adaptive contrast enhancement and de-enhancement," Pattern Recognition, vol. 24, no. 4 , pp. 289-302, 1991.

[12] N. C. Ho, V. N. Lan, and L. Xuan Viet, "Optimal hedgealgebras-based controller: Design and application," Fuzzy Sets and Systems, vol. 159, no. 8, pp. 968-989, 2008.

[13] J.-L. Starck, F. Murtagh, E. J. Candès, and D. L. Donoho, "Gray and color image contrast enhancement by the curvelet transform," IEEE Transactions on Image Processing, vol. 12, no. 6, pp. 706-717, 2003.

[14] C. H. Nguyen, D. K. Tran, H. Van Nam, and N. H. Chau, "Hedge algebras, linguistic-value logic and their application to fuzzy reasoning," International Journal of Uncertainty, Fuzziness and Knowledge-Based Systems, vol. 7, no. 04, pp. 347-361, 1999.

[15] J. C. Bezdek, Pattern recognition with fuzzy objective function algorithms. Springer Science \& Business Media, 2013.

[16] N. Ponomarenko et al., "Image database TID2013: peculiarities, results and perspectives," Signal Processing: Image Communication, vol. 30, pp. 57-77, 2015. 


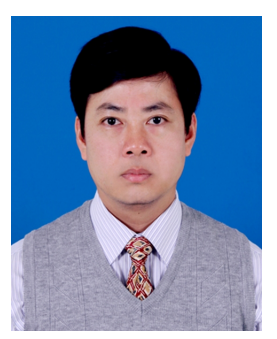

Nguyễn Văn Quyền sinh năm 1979 tại Hải Phòng. Ông nhận bằng Thạc sĩ, chuyên ngành Khoa học Máy tính tại Trường Đại học Sư phạm Hà Nội, năm 2008. Hiện nay, ông là Phó Trưởng phòng Phòng Quản lý Sau đại học, Trường Đại học Hải Phòng. Lĩnh vực nghiên cứu của ông là xử lý ảnh, khai phá dữ liệu và tính toán mềm.

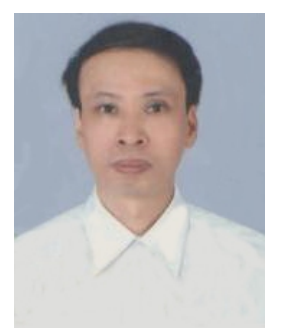

Ngô Hoàng Huy sinh năm 1969 tại Hà Nội. Ông nhận bằng Tiến sĩ, chuyên ngành Toán cho tin học, năm 2017, tại Học viện Khoa học và Công nghệ, Viện Hàn lâm Khoa học và Công nghệ Việt Nam. Hiện nay, ông đang là nghiên cứu viên tại Viện Công nghệ Thông tin, Viện Hàn lâm Khoa học và Công nghệ Việt Nam. Lĩnh vực nghiên cứu của ông là xử lý ảnh, xử lý tiếng nói, trí tuệ nhân tạo, mạng nơ ron học sâu, khai phá dữ liệu, hệ thống nhúng, IoT.

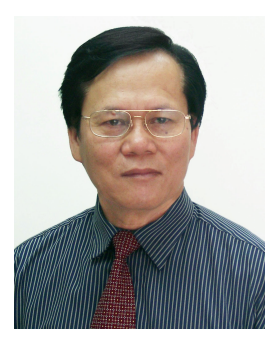

Nguyễn Cát Hồ sinh năm 1941 tại Hà Nội. Ông tốt nghiệp Trường Đại học Tổng hợp Hà Nội, năm 1960; nhận bằng Tiến sỹ tại Trường Đại học Tổng hợp Vacsava, Ba Lan, năm 1971 và nhận bằng Tiến sĩ Khoa học tại Trường Đại học Tổng hợp Kỹ thuật Dresden, Đức, năm 1987. Hiện nay, ông là cán bộ nghiên cứu, Trung tâm Nghiên cứu và Phát triển, Trường Đại học Duy Tân. Lĩnh vực nghiên cứu của ông là lô gic đại số, lô gic mờ, tính toán mềm, tính toán với từ, các hệ mờ và cơ sở dữ liệu mờ.

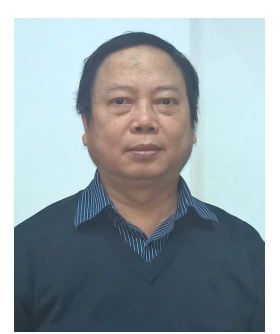

Trần Thái Sơn sinh năm 1955 tại Nghệ An. Ông nhận bằng Tiến sĩ, chuyên ngành Đảm bảo toán học cho máy tính và hệ thống tính toán, năm 1990, tại Trung tâm tính toán, Viện Hàn lâm Khoa học Liên Xô (cũ). Hiện nay, ông đang công tác tại Viện Công nghệ Thông tin, Viện Hàn lâm Khoa học và Công nghệ Việt Nam. Lĩnh vực nghiên cứu của ông là các hệ cơ sở dữ liệu, hệ chuyên gia và tính toán mềm. 